\title{
Kommunikation im Groves-Mechanismus - Ergebnisse eines Laborexperiments
}

\author{
Markus C. Arnold \\ Eva Ponick*
}

Oktober 2003

Aktuelle Version: 30. Januar 2004

\section{Zusammenfassung}

In der deutschsprachigen Literatur wird seit einigen Jahren der GrovesMechanismus als Anreizsystem zur wahrheitsgemäßen Berichterstattung intensiv diskutiert. Ein wichtiger Gesichtspunkt, der über die Anwendbarkeit des Groves-Mechanismus entscheidet, ist die Frage nach dessen Manipulationsresistenz. Bereichsmanager können nämlich bei diesem Entlohnungsmechanismus höhere Entlohnungen zu Lasten der Zentrale realisieren, wenn sie ihre Meldungen absprechen. Dies stellt jedoch im endlich wiederholten Fall spieltheoretisch kein Gleichgewicht dar.

Dieser Beitrag fasst die Ergebnisse eines Laborexperiments zusammen, bei dem die wahrheitsinduzierende Wirkung des Groves-Mechanismus untersucht wird. Dadurch, dass den Teilnehmern in einem von zwei Treatments die Möglichkeit gegeben wird, anonym miteinander zu kommunizieren, wird eine Verbindung zu den Gegebenheiten in der Praxis hergestellt. Kommunikation sollte theoretisch keine Auswirkung auf die Meldungen der Teilnehmer haben, da es sich dabei im spieltheoretischen Sinne um "cheap talk" handelt.

Die Ergebnisse zeigen, dass es weder im Fall mit noch im Fall ohne Kommunikation im Groves-Mechanismus zu wahrheitsgemäßer Berichterstattung kommt. Zusätzlich führt die anonyme Kommunikation zu stabilen Kooperationen zwischen den Teilnehmern. Die durchschnittlichen Abweichungen von der wahrheitsgemäßen Berichterstattung steigen für diesen Fall im Verlauf des Experiments sogar an.

Führt der Groves-Mechanismus aber in keinem der Treatments zur wahrheitsgemäßen Berichterstattung und selbst bei dieser eingeschränkten Form der Kommunikation gar zu stabilen Manipulationsstrategien der Teilnehmer, erstaunt es nicht, dass dieser Mechanismus trotz seiner wünschenswerten theoretischen Eigenschaften in der Praxis nicht angewendet wird.

\footnotetext{
*Dipl.-Kfm. Markus Arnold und Dipl.-Math. Eva Ponick, Technische Universität Clausthal, Institut für Wirtschaftswissenschaft, Julius-Albert-Str. 2, 38678 Clausthal-Zellerfeld.

Wir danken Heike Y. Schenk-Mathes für viele hilfreiche Anregungen und Kommentare und Stefan Geisler für die Unterstützung bei der technischen Umsetzung des Experiments.
} 


\section{Kommunikation im Groves-Mechanismus - Ergebnisse eines Laborexperiments}

\section{Einleitung}

Die Rahmenbedingungen, innerhalb derer Unternehmen agieren, sind in den vergangenen Jahren zunehmend komplexer geworden und haben zu einer steigenden Dezentralisierung von Entscheidungen im Unternehmen und damit zur Bildung so genannter Investment und Profit Center geführt. Dies macht es zum einen möglich, das Spezialwissen der dezentralen Abteilungen für den Entscheidungsprozess zu nutzen, und ermöglicht zum anderen eine stärkere Fokussierung der Zentrale auf strategische Aufgaben. Jedoch ergibt sich ebenfalls die Notwendigkeit zur zentralen Koordination der dezentralen Einheiten zumindest aufgrund von möglicherweise bestehenden sachlichen Interdependenzen zwischen den einzelnen Teilbereichen im Unternehmen. ${ }^{1}$ Eine der grundlegenden Methoden, um diese Interdependenzen zu koordinieren, stellt die Budgetierung dar.

Gerade der traditionelle Budgetierungsprozess im Unternehmen wird in letzter Zeit allerdings stark kritisiert, da er für Manager in den Budgetverhandlungen offensichtlich einen Anreiz setzt, die Unwahrheit zu sagen, was die Fehlallokation von Ressourcen innerhalb des Unternehmens und damit Wertvernichtung für die Eigentümer nach sich ziehen kann. ${ }^{2}$ Um solche verzerrten Meldungen über die Gewinnpotentiale der einzelnen Unternehmensbereiche zu vermeiden, wurde jedoch in der Vergangenheit bereits eine Reihe von wahrheitsinduzierenden Entlohnungsmechanismen konstruiert. ${ }^{3}$

$\mathrm{Zu}$ den bekanntesten zählt dabei eine Klasse von Entlohnungsmechanismen, die von Vickrey (1964), Clarke (1971) und Groves (1973) entwickelt und von Groves/Loeb (1979) auf die Budgetierungsproblematik in Unternehmen erweitert wurde. Bei dem in diesem Kontext verwendeten Mechanismus ist die variable Vergütung eines Bereichsmanagers abhängig vom eigenen tatsächlichen Gewinn und den gemeldeten Gewinnen der anderen Bereichsmanager. In der deutschsprachigen Literatur wird das üblicherweise als Groves-Mechanismus bezeichnete Entlohnungsschema seitdem intensiv diskutiert ${ }^{4}$ und dabei insbesondere der Frage nachgegangen, warum der Groves-Mechanismus trotz seiner theoretisch äußerst wünschenswerten Eigenschaften keine Anwendung in der Praxis findet.

Einer der beiden wesentlichen Kritikpunkte, die sich aus den theoretischen Beiträgen herauskristallisieren und mögliche Erklärungen für die fehlende Anwendung des Groves-Mechanismus in Budgetierungsprozessen von Unternehmen bieten könnten, ist, dass die Bereichsmanager durch Absprachen ihre Entlohnungen zu Lasten der Instanz erhöhen können. ${ }^{5}$ Andererseits wird die mangelnde Effizienz des GrovesMechanismus kritisiert, wenn neben das Problem der asymmetrischen Informationsverteilung zwischen Bereichsmanager und Zentrale auch noch ein moral hazardProblem tritt. ${ }^{6}$ Der Groves-Mechanismus lässt nämlich keine Abwägung zwischen

\footnotetext{
${ }^{1}$ Vgl. Ewert/Wagenhofer (2000), S. 446-449, Laux/Liermann (2003), Kapitel XII.

${ }^{2}$ Vgl. Jensen (2001a,b).

${ }^{3}$ Vgl. z.B. Weitzman (1976), Reichelstein/Osband (1984), Osband/Reichelstein (1985).

${ }^{4}$ Vgl. Bamberg/Locarek (1992), Locarek/Bamberg (1994), Arbeitskreis "Finanzierung" (1994), Pfaff/Leuz (1995), Budde/Göx/Luhmer (1998), Kunz/Pfeiffer (1999), Hofmann/Pfeiffer (2003).

${ }^{5}$ Vgl. u.a. Banker/Datar (1992), Pfaff/Leuz (1995), S. 673, und besonders Kunz/Pfeiffer (1999).

${ }^{6}$ Vgl. Hofmann/Pfeiffer (2003). Pfaff/Leuz (1995) zeigen in diesem Zusammenhang, dass der Groves-Mechanismus bei Risikoaversion der Bereichsmanager ineffiziente Risikoverteilung induziert (S. 676-677) und unter bestimmten Umständen sogar seine wahrheitsinduzierende Wirkung
} 
dem Informationsproblem auf der einen und dem Anreizproblem auf der anderen Seite zu.

Bezogen auf den ersten und für diesen Beitrag relevanten Kritikpunkt lässt sich tatsächlich zeigen, dass Bereichsmanager höhere Entlohnungen als im Fall der wahrheitsgemäßen Berichterstattung realisieren können, wenn sie ihre Meldungen absprechen und höhere als ihre tatsächlichen Produktivitäten bzw. Gewinnfunktionen melden. Dies stellt aber bei individuell rationalem Verhalten aller Bereichsmanager im spieltheoretischen Sinne kein Gleichgewicht dar, da gegeben die Meldung der anderen jeder Bereichsmanager einen Anreiz hat, von seiner Übertreibung abzuweichen und wahrheitsgemäß zu berichten. ${ }^{7}$ Jedoch kann es beim Groves-Mechanismus zu Kooperation, also zu überhöhten Meldungen der Gewinnpotentiale kommen, wenn Unsicherheit darüber besteht, ob die anderen Bereichsmanager individuell rational oder kooperativ im Sinne einer Tit-for-tat-Strategie handeln. ${ }^{8}$ Die Kooperation, die auf dem Aufbau einer Reputation als kooperativer Spieler beruht, nimmt jedoch im Laufe des Spiels ab.

Auf den zweiten Kritikpunkt, dass der Groves-Mechanismus bei Risikoaversion der Bereichsmanager und Erweiterung der Problemstellung um ein moral hazardProblem keine optimalen Ergebnisse mehr liefert, soll hier nicht genauer eingegangen werden. Budde/Göx/Luhmer (1998) argumentieren nämlich, dass eine solche Problematik beispielsweise nicht auf den Fall einer "zweigleisigen Unternehmensführung" zutrifft, bei der der Bericht an die Zentrale über die Gewinnpotentiale eines Bereichs einem Controller und nicht mehr dem Bereichsmanager überlassen bleibt. ${ }^{9}$ Jedoch bliebe die Frage nach einer möglichen Manipulationsstrategie zwischen den Controllern erhalten, "weil die Controller der verschiedenen Abteilungen rollenbedingt zur Zusammenarbeit neigen werden." 10

Dies ist die Problemstellung, die auch in dem hier vorgestellten Experiment untersucht werden soll. Würde sich ergeben, dass der Groves-Mechanismus selbst in einem solchen Fall ohne zusätzliche Motivationsproblematik nicht zu wahrheitsgemäßer Berichterstattung führt, könnte dies einen deutlichen Hinweis auf seine fehlende Präsenz in der Praxis liefern, denn dann würde er noch nicht einmal seine grundlegende Funktion der Induzierung wahrheitsgemäßer Meldungen erfüllen.

Die wahrheitsinduzierende Wirkung des Groves-Mechanismus experimentell zu überprüfen ist umso wichtiger, als die experimentelle Forschung bisher gezeigt hat, dass sich Experimentteilnehmer keinesfalls alle so verhalten, wie es unter standardtheoretischen Annahmen abgeleitet wurde. Die Anzahl der experimentellen Ergebnisse zum Groves-Mechanismus ist dabei bisher vergleichsweise gering. Waller/Bishop (1990) untersuchen die wahrheitsinduzierende Wirkung mehrerer Entlohnungsmechanismen, darunter des Groves-Mechanismus, und finden bei diesem Entlohnungsschema nur $48 \%$ wahrheitsgemäße Berichterstattung. Offensichtlich gelingt aber auch nur in den seltensten Fällen eine Kooperation zwischen den Spielern. ${ }^{11}$ Chow/Hirst/Shields $(1994,1995)$ finden zum einen, dass bei der wahrheitsgemäßen Berichterstattung der Groves-Mechanismus besser abschneidet als eine alleinige Beteiligung am Bereichsgewinn, zum anderen jedoch, dass eine Bereichsgewinnbeteiligung plus zusätzlicher Möglichkeit zu kostenlosen und perfekten Audits, die zufällig die gemeldeten Produktivitäten überprüfen, einen signifikant höheren Anteil wahrheitsgemäßer Berichterstattungen erzeugt als der Groves-Mechanismus. Chow/Hwang/Liao (2000) vergleichen den Groves-Mechanismus mit einer Bereichs-

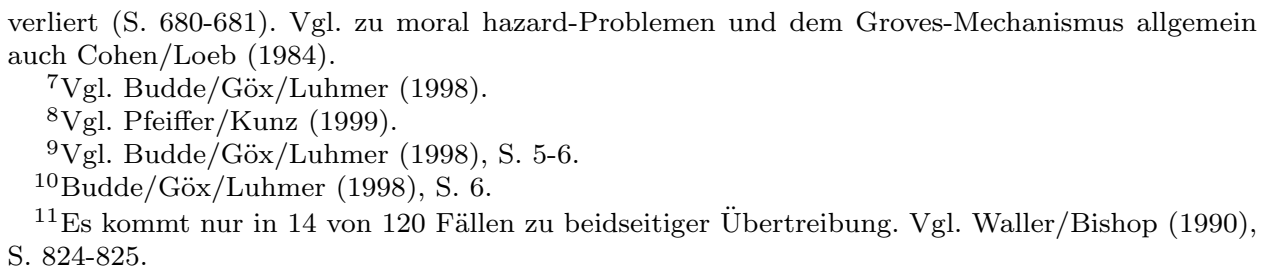

${ }^{11}$ Es kommt nur in 14 von 120 Fällen zu beidseitiger Übertreibung. Vgl. Waller/Bishop (1990), S. 824-825. 
gewinnbeteiligung, bei der die Zentrale zusätzlich Audits durchführen kann, die Kosten verursachen und keine perfekten Ergebnisse liefern, und in Abhängigkeit des Ergebnisses diskretionär über die Annahme von Projekten entscheiden kann. Sie erhalten bei letzterem Anreizschema signifikant weniger Abweichungen von der wahrheitsgemäßen Berichterstattung als beim Groves-Mechanismus. Wie in Waller/Bishop (1990) sind jedoch ebenfalls in jedem der drei letztgenannten Experimente die Anteile der wahrheitsgemäßen Berichterstattung bei Entlohnung gemäß dem Groves-Mechanismus vergleichsweise gering und liegen nur zwischen $32 \%$ und $40 \%$. Ebenfalls erstaunlich ist, dass wie Waller/Bishop (1990) auch Chow/Hirst/Shields (1994) und Chow/Hwang/Liao (2000) keine Anzeichen dafür finden, dass die unwahrheitsgemäße Berichterstattung beim Groves-Mechanismus auf kollusives Verhalten der Teilnehmer zu Lasten der Instanz zurückzuführen ist.

Das hier vorgestellte Experiment ähnelt in der Struktur dem von Waller/Bishop (1990), ermöglicht in einem Treatment jedoch auch (anonyme) Kommunikation zwischen den jeweiligen Spielpartnern vor Abgabe der Meldung. Damit soll eine Annäherung an die Gegebenheiten der Praxis geschaffen werden, wo sicherlich sogar noch weitergehende Kommunikationsmöglichkeiten als die hier untersuchte bestehen. Ebenso wie in der Praxis sind bindende Verträge über die Manipulationsstrategien zwischen den Spielpartnern allerdings nicht möglich.

Die Hauptergebnisse des Beitrags sind folgende: In Übereinstimmung mit den Ergebnissen früherer Experimente führt der Groves-Mechanismus weder im Treatment mit noch ohne Kommunikation mehrheitlich zu wahrheitsgemäßer Berichterstattung der Experimentteilnehmer. Nur in $44 \%$ aller Fälle ohne Kommunikation bzw. $22 \%$ der Fälle mit Kommunikation werden die Produktivitäten wahrheitsgemäß gemeldet. Während jedoch die Abweichungen im Treatment ohne Kommunikation auf Probleme beim Verständnis des Groves-Mechanismus und soziale Präferenzen zurückgeführt werden können, sind die Abweichungen bei Kommunikation Ergebnis koordinierter Manipulationsstrategien. Es zeigt sich, dass sich die Meldestrategien der Teilnehmer in beiden Treatments signifikant unterscheiden. Obwohl die theoretischen Ergebnisse zumindest ein Absinken der Manipulationsbeträge in den letzten Runden vorhersagen, ergibt sich dies im Experiment in keinem der beiden Treatments. Bei Kommunikation steigen die Manipulationsbeträge während den ersten Runden sogar an, und es kommt im Anschluss zu stabilen Kooperationsstrategien der Teilnehmer. Offensichtlich führt bereits die Möglichkeit zur anonymen Kommunikation dazu, dass sich beim Groves-Mechanismus nun tatsächlich (stabiles) kollusives Verhalten der Teilnehmer zu Lasten der Instanz ergibt, was bezogen auf die Gegebenheiten der Praxis mit ihren vielfältigen Kommunikationsmöglichkeiten einen sehr starken Hinweis auf seine fehlende Verwendung in Unternehmen liefert.

Dieser Beitrag liefert damit ebenfalls weitere Erkenntnisse darüber, wie sich Kommunikation auf Problemstellungen der Spieltheorie auswirkt. Wie bei bisherigen Untersuchungen zeigt sich auch hier, dass Kommunikation offensichtlich für reale Entscheidungssituationen mehr als nur "cheap talk" im Sinne der Spieltheorie darstellt. ${ }^{12}$

Ebenso erweitert dieser Beitrag die experimentelle Forschung im Bereich der wiederholten Gefangenendilemmata, wobei hier im Gegensatz zu den üblicherweise verwendeten Public Good-Spielen oder ähnlichen vergleichsweise einfachen Spielstrukturen der als verhältnismäßig komplex einzustufende Groves-Mechanismus zur Anwendung kommt.

Der Beitrag ist wie folgt gegliedert: In Abschnitt 2 werden zunächst aus den theoretischen Überlegungen die Hypothesen für das Experiment abgeleitet. Abschnitt 3

\footnotetext{
${ }^{12}$ Vgl. u.a. Isaac/McCue/Plott (1985), Isaac/Walker(1988), Brosig/Weimann/Ockenfels (2003), Bolton/Chatterjee/McGinn (2003).
} 
beschreibt den Aufbau des Experiments. Danach werden in Abschnitt 4 zunächst die generellen Ergebnisse des Experiments vorgestellt, während Abschnitt 5 die Auswirkungen der Kommunikation im Detail untersucht. Der Beitrag schließt in Abschnitt 6 mit einer Zusammenfassung der Ergebnisse und den Schlussfolgerungen, die daraus zu ziehen sind.

\section{Ableitung der Hypothesen}

Groves/Loeb (1979) untersuchen die Problematik der Ressourcenallokation in einem Unternehmen mit mehreren Divisionen und übertragen den Groves-Mechanismus als Anreizschema zur wahrheitsgemäßen Berichterstattung auf den Budgetierungsprozess in Unternehmen. Bei diesem Entlohnungsmechanismus ist die Entlohnung eines Bereichsmanagers eine steigende Funktion des eigenen tatsächlichen Bereichsgewinns und der gemeldeten Gewinne der anderen Bereiche, die sich auf Basis der an die Zentrale gemeldeten Produktions- bzw. Gewinnfunktionen berechnen.

Aufgrund dieser Beteiligung an den gemeldeten Gewinnen der anderen Bereiche wird jedoch in der Literatur häufig kritisiert, dass sich die Manager durch Absprachen und überhöhte Meldungen ihrer Gewinnpotentiale zu Lasten der Instanz bereichern können. ${ }^{13}$ Folglich würde der Groves-Mechanismus auch nicht zu wahrheitsgemäßer Berichterstattung führen.

Aufbauend auf dieser Kritik zeigen Budde/Göx/Luhmer (1998) dagegen, dass diese Manipulationsmöglichkeit zwar existiert, jedoch keine Gleichgewichtsstrategie für die Manager darstellt. Gegeben die Meldung der anderen Manager ist es im Einperiodenfall für jeden Manager individuell rational, von seiner Übertreibung abzuweichen und sein Gewinnpotential wahrheitsgemäß zu melden: Es ergibt sich das entsprechende Gleichgewicht in dominanten Strategien. ${ }^{14}$ Diese Lösung lässt sich per Rückwärtsinduktion direkt auf den endlich wiederholten Fall übertragen. Die Manipulation im Groves-Mechanismus spiegelt somit das klassische Problem eines Gefangenendilemmas der Bereichsmanager wider. Auf diesem endlich wiederholten Fall beruht auch das vorliegende Experiment, wobei die Anzahl der Spielrunden den Teilnehmern bekannt war. Würden sich also alle Spieler individuell rational verhalten, müsste der Groves-Mechanismus in diesem Experiment ab der ersten Runde zur wahrheitsgemäßen Berichterstattung führen.

Nimmt man jedoch an, dass für die Spieler Unsicherheit darüber besteht, ob der Gegenspieler individuell rational oder kooperativ im Sinne einer Tit-for-tatStrategie spielt, weisen Kreps/Milgrom/Roberts/Wilson (1982) nach, dass es im endlich wiederholten Gefangenendilemma auch für individuell rationale Spieler vorteilhaft sein kann, kooperativ zu spielen. Dies führt zum Aufbau einer dementsprechenden Reputation und zur Realisation höherer Entlohnungen. ${ }^{15}$ Kunz/Pfeiffer (1999) übertragen dies auf den Groves-Mechanismus und zeigen, dass es auch hier im endlich wiederholten Fall phasenweise zu Kooperation zwischen individuell rationalen Spielern und somit zu überhöhten Meldungen der Gewinnpotentiale kommen kann. Allerdings nimmt diese Kooperation im Verlauf des Spiels ab, da die Vorteilhaftigkeit zu defektieren mit zunehmender Spieldauer ansteigt. Dies wird vor allem

\footnotetext{
${ }^{13}$ Vgl. Banker/Datar (1992), Ewert/Wagenhofer (2000), Pfaff/Leuz (1995), Laux (1999).

${ }^{14}$ Vgl. Budde/Göx/Luhmer (1998), S. 17, auch Laux (1999), S. 543, Ewert/Wagenhofer (2000), S. 563-565.

${ }^{15}$ Diese Argumentation dient oftmals als Basis zur Erklärung des kooperativen Verhaltens von Spielern in Experimenten zu endlich wiederholten Gefangenendilemmata. Krapp (2000) zeigt, dass auch bei einer Simulation, in der verschiedene Strategien gegeneinander antreten, im Fall ohne Diskontierung die Reputationsstrategie sehr gut abschneidet. Vgl. zu experimentellen Überprüfungen der Reputationshypothese Camerer/Weigelt (1988), Andreoni/Miller (1993), Cooper/DeJong/Forsythe/Ross (1996).
} 
an der letzten Spielrunde deutlich: Da es hier nicht mehr sinnvoll sein kann, eine Reputation als kooperativer Spieler aufzubauen, werden spätestens dann alle individuell rationalen Spieler ihre Gewinnpotentiale wahrheitsgemäß melden. Es sollte also ein Endrundeneffekt auftreten, der auch häufig in Experimenten zu wiederholten Gefangenendilemmata beobachtet werden kann. ${ }^{16}$

Aufgrund dieser unterschiedlichen theoretischen Ergebnisse werden für das Verhalten der Experimentteilnehmer folgende - sich gegenseitig ausschließende - Hypothesen aufgestellt:

Hypothese 1a: Der Groves-Mechanismus führt ab der ersten Runde zu wahrheitsgemäßer Berichterstattung der Teilnehmer.

Hypothese 1b: Kommt es zu Abweichungen von der wahrheitsgemäßen Berichterstattung, nehmen diese mit zunehmender Rundenzahl ab und tendieren gegen null.

In dem hier vorgestellten Experiment wurde den Teilnehmern zudem in einem Treatment vor Abgabe der Meldung die Möglichkeit zur anonymen Kommunikation eingeräumt. Da die Teilnehmer sich zum einen nicht identifizieren konnten und zum anderen keine bindenden Verträge über ihre Manipulationsstrategien abschließen konnten, handelt es sich bei der Kommunikation im spieltheoretischen Sinne um "cheap talk". Aus diesem Grund ergibt sich folgende Hypothese bezüglich der Auswirkungen der Kommunikation:

Hypothese 2: Die Möglichkeit zur Kommunikation hat keinen Einfluss auf die Meldungen der Teilnehmer.

Diese Hypothese berücksichtigt ausschließlich die spieltheoretischen Vorhersagen für diese Situation. Experimentelle Studien zeigen jedoch, dass Kommunikation die Ergebnisse von Experimenten in nicht unerheblicher Weise beeinflusst, selbst wenn sie wie hier nur "cheap talk" darstellt. Insbesondere bei wiederholten Gefangenendilemmata kann Kommunikation zu erhöhter Kooperation führen. ${ }^{17}$ Mangels theoretischer Fundierung der Auswirkungen der Kommunikation auf das Verhalten der Experimentteilnehmer werden diese experimentellen Ergebnisse bei der Formulierung von Hypothese 2 jedoch nicht berücksichtigt.

\section{Aufbau des Experiments}

Das Experiment zum Groves-Mechanismus bestand für alle Teilnehmer zunächst aus 12 Proberunden, in denen sie die Funktionsweise des Mechanismus kennen lernen und die Auswirkungen unterschiedlicher Meldestrategien selbst testen konnten. An die Proberunden schlossen sich 10 Spielrunden an, die ausschließlich für die Entlohnung der Teilnehmer relevant waren. Zu Beginn der Spielrunden wurde jedem Experimentteilnehmer für die gesamte Dauer der 10 Runden ein Mitspieler zugeteilt. Den Teilnehmern waren sowohl die Anzahl der Spielrunden als auch die Tatsache bekannt, dass ihr Spielpartner während dieser 10 Runden der gleiche bleiben würde.

\footnotetext{
${ }^{16}$ Vgl. zu den Ergebnissen in Experimenten zum wiederholten Gefangenendilemma z.B. Davis/Holt (1993), S. 317-343, Ledyard (1995). Auch im Fall eines Public Good-Spiels mit Kommunikation ist dieser negative Zeittrend zu beobachten. Vgl. Brosig/Weimann/Ockenfels (2003).

${ }^{17}$ Vgl. Isaac/McCue/Plott (1985), Isaac/Walker(1988), Bolton/Chatterjee/McGinn (2003). Brosig/Weimann/Ockenfels (2003) zeigen, dass das Kooperationsniveau bei zunehmenden Kommunikationsmöglichkeiten ebenfalls ansteigt. Hannan/Rankin/Towry (2003) untersuchen zwar keine Kommunikation, finden aber in ihrem Experiment, dass im Budgetierungsprozess die wahrheitsgemäße Berichterstattung ansteigt, wenn Manager die Information persönlich und nicht anonym übermitteln, was ebenfalls nicht mit standardtheoretischen Annahmen zu erklären ist.
} 
Das Experiment wurde in zwei unterschiedlichen Varianten gespielt, wobei die Spieler jeweils nur an einer der beiden Varianten teilnahmen: Im Treatment mit Kommunikation konnten die Teilnehmer vor Abgabe ihrer Meldung an die zentrale Instanz mit ihrem Spielpartner mittels eines Chatprogrammes kommunizieren, während dies im Treatment ohne Kommunikation nicht möglich war. Auf die genaue Form der Kommunikation soll später eingegangen werden.

Der allgemeine Aufbau des Experiments ähnelt von der Struktur dem Experiment von Waller/Bishop (1990). In dem hier durchgeführten Experiment werden die Ressourceneinheiten von einer zentralen Instanz zwischen zwei konkurrierenden Unternehmensbereichen aufgeteilt. Dabei besteht asymmetrische Informationsverteilung in dem Sinne, dass die Teilbereiche im Zeitpunkt der Zuteilung der Ressource über die Gegebenheiten in ihrem jeweiligen Bereich, insbesondere die entsprechenden Produktivitätsparameter besser informiert sind als die Zentrale. Diese möchte die Ressource so zwischen den beiden Bereichen aufteilen, dass der Unternehmensgesamtgewinn maximiert wird, der sich hier als Summe der beiden Bereichsgewinne ergibt. Aus diesem Grund ist sie daran interessiert, dass ihr die fehlenden Informationen von den Bereichsmanagern wahrheitsgemäß übermittelt werden.

Dies konkretisiert sich hier in folgender Weise. Für die in den Bereichen vorliegenden Gewinnfunktionen $G_{i}\left(v_{i}\right)$ gilt:

$$
\begin{aligned}
G_{i}\left(v_{i}\right) & = \begin{cases}p_{i} \cdot v_{i}-v_{i}=\left(p_{i}-1\right) \cdot v_{i} & \text { für } v_{i} \leq 40 \\
40 \cdot p_{i}+\left(v_{i}-40\right) \cdot\left(p_{i}-0,3\right)-v_{i} & \text { für } 40<v_{i} \leq 100 \\
40 \cdot p_{i}+60 \cdot\left(p_{i}-0,3\right)+\left(v_{i}-100\right)-v_{i} & \text { für } 100<v_{i}\end{cases} \\
\forall i & =1,2
\end{aligned}
$$

wobei $p_{i} \in\{1,4 ; 1,5 ; \ldots ; 2,1\}$ die Produktivität des Bereichs $i$ und $v_{i}$ die dem Bereich $i$ zugeteilten Ressourceneinheiten darstellen. Die ersten 40 Ressourceneinheiten, die den Bereichen zugeteilt werden, können also zu der tatsächlichen ursprünglichen Produktivität $p_{i}$ angelegt werden, darüber hinausgehende Ressourceneinheiten dagegen nur noch zu einer Produktivität, die um 0,3 geringer ist als die ursprüngliche. Alle Ressourceneinheiten, die über 100 hinausgehen, können schließlich nicht mehr produktiv eingesetzt werden. Da für die Produktivitäten beider Bereiche in jeder Runde $p_{i} \geq 1,4$ gilt, könnten die Bereiche insgesamt 200 Einheiten der Ressource produktiv einsetzen. Der zentralen Instanz stehen dagegen nur maximal $\bar{V}=120$ Ressourceneinheiten zur Verfügung, die Ressource ist somit knapp. Die allgemeine Gestalt der Gewinnfunktion beider Bereiche ist der zentralen Instanz zwar bekannt, jedoch kennen nur die Bereichsmanager in jeder Spielrunde ihre jeweiligen Produktivitäten $p_{i}$.

Im Vergleich zur Gewinnfunktion von Waller/Bishop (1990) weisen die hier verwendeten Gewinnfunktionen je nach Anzahl der eingesetzten Ressourceneinheiten unterschiedliche positive Grenzproduktivitäten auf. Somit ist es nicht optimal, dem Bereich mit der höheren Produktivität stets das Maximum an Ressourcen zuzuteilen, sondern es ergibt sich in Abhängigkeit der Bereichsproduktivitäten für $p_{j} \geq p_{i}$ folgende optimale Ressourcenaufteilung:

$$
\begin{array}{lll}
v_{i}=20 \text { und } v_{j}=100 & \forall p_{i}<p_{j}-0,3 \\
v_{i}=40 \text { und } v_{j}=80 & \forall p_{j}-0,3 \leq p_{i}<p_{j} \\
v_{i}=v_{j}=60 & \forall p_{i}=p_{j}
\end{array}
$$

Entsprechendes gilt für $p_{i} \geq p_{j}$. Das bedeutet, dass beide Bereiche nur dann die gleiche Anzahl Ressourceneinheiten erhalten sollten, wenn sie auch die gleiche Produktivität haben. Besitzt dagegen ein Bereich eine höhere Produktivität als der andere, sollten diesem Bereich mehr Ressourcen zugewiesen werden, damit der Gesamtgewinn maximiert wird. Je nach Differenz ist es dabei optimal, dem "produktiveren" Bereich entweder 80 oder gar 100 Ressourceneinheiten zuzuteilen. 
Die Beteiligung eines Bereichsmanagers alleine an seinem eigenen Bereichsgewinn führt dann bei der Meldung der Produktivität zu Übertreibungen, da er stets 100 Ressourceneinheiten produktiv einsetzen kann und somit den eigenen Bereichsgewinn bei $v_{i}=100$ maximiert. Beide Bereichsmanager würden also stets $\widehat{p}_{i}=\widehat{p}_{j}=2,1$ melden, um sich zumindest 60 Ressourceneinheiten zu sichern. Zur Induzierung einer wahrheitsgemäßen Berichterstattung werden die Bereichsmanager in dem hier vorgestellten Rahmen deshalb gemäß dem Groves-Mechanismus entlohnt.

Für die Entlohnung der Bereichsmanager pro Runde wurde im Experiment folgende Funktion gewählt:

$$
P_{i}=0,1 \cdot\left[G_{i}\left(\widehat{v}_{i}\right)+\widehat{G}_{j}\left(\widehat{v}_{j}\right)\right]
$$

wobei $P_{i}$ die erreichte Anzahl Punkte pro Runde angibt, $\widehat{v}_{i}$ und $\widehat{v}_{j}$ die den beiden Bereichen aufgrund ihrer Meldungen zugeteilten Ressourceneinheiten, $G_{i}\left(\widehat{v}_{i}\right)$ den tatsächlichen Bereichsgewinn des Bereichs $i$ auf Basis von $\widehat{v}_{i}$ und $\widehat{G}_{j}\left(\widehat{v}_{j}\right)$ den gemeldeten Bereichsgewinn des Bereichs $j$ auf Basis von $\widehat{v}_{j}$. Die gesamte variable Entlohnung des Experiments in Euro berechnete sich auf Basis der Summe der in den 10 Runden erreichten Punkte.

Dass hier - wie bereits erwähnt - die wahrheitsgemäße Berichterstattung die dominante Meldestrategie darstellt, soll anhand des Beispiels in Tabelle 1 aufgezeigt werden. Es ist zu erkennen, dass zwar nicht jede Abweichung von der wahrheitsgemäßen Meldung zu einer Verringerung der Entlohnung führt, dass die Entlohnung bei Meldung der tatsächlichen Produktivität von $p_{i}=1,8$ jedoch in mindestens einer Konstellation strikt höher ist als bei Meldung jeder anderen Produktivität. Unter der Annahme, dass in der Tabelle 1 auch für Spieler $j$ eine tatsächliche Produktivität von $p_{j}=1,8$ gilt, zeigt diese Tabelle auch das Gefangenendilemma auf, in dem sich beide Spieler bei Abgabe ihrer Meldung befinden: Könnten beide Spieler bindende Absprachen treffen, wäre es für sie vorteilhaft, ihre Meldung abzusprechen und $\widehat{p}_{i}=\widehat{p}_{j}=2,1$ zu melden. Da der Abschluss bindender Verträge mit dem Ziel der Manipulation jedoch weder in der Realität möglich sein dürfte, noch hier im Experiment in irgendeinem der Treatments möglich war, ist es gegeben die Übertreibung (bzw. allgemein die Meldung) des Mitspielers für jeden Spieler wieder individuell rational, seine tatsächliche Produktivität von $p_{i}=p_{j}=1,8$ zu melden. Im Beispiel von Tabelle 1 würde sich die Bemessungsgrundlage dann von 102 auf 108 erhöhen, falls Spieler i die Wahrheit meldet und Spieler j weiterhin übertreibt.

Die Struktur des Experiments entspricht somit der eines endlich wiederholten Gefangenendilemmas, wobei jedoch anstelle eines simplen Public Good-Spiels der komplexere Groves-Mechanismus gespielt wird, was u.a. impliziert, dass jeder Spieler aus der Meldung seines Mitspielers nicht direkt auf dessen Kooperationswillen schließen kann. Selbst eine Meldung von $\widehat{p}_{i}=2,1$ kann nämlich einfach der rational eigennützigen Strategie entsprechen, wenn der Spieler zufällig in dieser Runde eine tatsächliche Produktivität in eben dieser Höhe besitzt. Auf diese Unterschiede und ihre Auswirkungen soll später noch genauer eingegangen werden.

Besäßen die Spieler die Möglichkeit, sich ex ante verbindlich auf Meldestrategien zu einigen, bestünde die optimale Strategie unter den hier vorgestellten Rahmenbedingungen im Fall ohne Kommunikation darin, stets $\widehat{p}_{i}=\widehat{p}_{j}=2,1$ zu melden, da dies für beide Spieler in jeder Runde zu einer erwarteten Bemessungsgrundlage von $E\left(B G_{i}\right)=99$ führt. Wäre beiden Spieler vor Abgabe ihrer Meldung die tatsächliche Produktivität des Mitspielers bekannt und könnten sie sich wiederum ex ante glaubhaft an eine Strategie binden, wäre es dagegen von Vorteil, dass der Spieler mit der niedrigeren Produktivität eine Meldung von $\widehat{p}_{i}=2,0$ und der mit der höheren Produktivitä eine von $\widehat{p}_{j}=2,1$ abgibt. Dies würde zu einem Erwartungswert der Bemessungsgrundlage in jeder Runde in Höhe von $E\left(B G_{i}\right)=99,88$ führen. Wie 
bereits oben erläutert, stellen diese Strategien jedoch im spieltheoretischen Sinne kein Gleichgewicht dar, da die Spieler keine Möglichkeit haben, sich verbindlich zu einigen.

In den Instruktionen zum Experiment wurde den Teilnehmern die Ermittlung der Bereichsgewinne gemäß (1), die Aufteilung der Ressource gemäß (2) und ihre Entlohnung gemäß (3) erläutert. ${ }^{18}$ Die Instruktionen konnten von den Teilnehmern von Computerbildschirmen abgelesen werden und wurden gleichzeitig laut vorgelesen. Jeder Teilnehmer durfte jeden Bildschirm solange einsehen, wie er wollte. Der genaue Wortlaut der Instruktionen ist dem Anhang zu entnehmen.

Um die Funktionsweise des Groves-Mechanismus zu verstehen, wurde den Teilnehmern die Möglichkeit gegeben, 12 Proberunden zu spielen. ${ }^{19}$ In jeder Proberunde waren die eigene tatsächliche Produktivität sowie die Meldung eines simulierten Spielpartners gegeben. Für jeweils drei Proberunden wurde die Kombination dieser beiden Produktivitäten konstant gehalten. Die Experimentteilnehmer hatten also theoretisch die Möglichkeit, bei jeder Kombination die Auswirkungen von Über-, Untertreibung und wahrheitsgemäßer Berichterstattung zu testen. ${ }^{20}$ Die genauen Werte der Proberunden sind ebenfalls den Instruktionen im Anhang zu entnehmen.

Zusammenfassungen der Formeln zur Ermittlung des Bereichsgewinns, der Entlohnung und der zugeteilten Ressourceneinheiten wurden den Spielern am Ende der Instruktionen und vor Beginn der Proberunden ausgeteilt. Zusätzlich wurden der Kommunikationsgruppe vor Beginn der 10 Spielrunden auch noch Kommunikationsregeln ausgeteilt. Auch diese sind im Anhang aufgeführt.

Das Treatment mit Kommunikation unterscheidet sich von dem Treatment ohne dadurch, dass den Spielern die Möglichkeit gegeben wurde, nach Kenntnisnahme der eigenen tatsächlichen Produktivität für die Runde und vor Abgabe der Meldung an die zentrale Instanz mittels eines Chatprogrammes mit ihrem Mitspieler zu kommunizieren. Die Dauer der Chatphase betrug in der ersten Periode 4 Minuten und reduzierte sich im Laufe der 10 Spielrunden auf 2 Minuten. Die Kommunikation war anonym, kein Spieler konnte in irgendeiner Weise auf die Identität seines Mitspielers schließen. Zudem wurde den Spielern unter Androhung des Verlustes der gesamten variablen Entlohnung der 10 Spielrunden explizit verboten, sich in irgendeiner Weise zu identifizieren oder zu versuchen, Verabredungen zu treffen, die über das Spiel im Labor hinausreichten. ${ }^{21}$ Bindende Absprachen waren also zwischen den Spielern zu keinem Zeitpunkt möglich.

Bei Abgabe der eigenen Meldung war in beiden Treatments jedem Spieler nur die eigene tatsächliche Produktivität bekannt, nicht aber die Meldung oder die tatsächliche Produktivität des Spielpartners. Die Meldung des Spielpartners wurde ihnen am Ende der Runde bei der Auswertung bzw. der Bekanntgabe der Höhe der Entlohnung für die entsprechende Runde mitgeteilt, da sie diese ohnehin aus der eigenen Entlohnung, in die eben auch der nicht mit Unsicherheit behaftete gemeldete Gewinn des Partners einging, hätten errechnen können. Die tatsächliche Produktivität des Partners wurde den Experimentteilnehmern aber auch zu diesem

\footnotetext{
${ }^{18}$ Den Spielern wurde jedoch zu keinem Zeitpunkt mitgeteilt, dass es sich bei der Aufteilung der Ressource gemäß (2) um die für die zentrale Instanz optimale Allokation handelt.

${ }^{19}$ Die insgesamt 22 Runden, die jeder Teilnehmer spielte, boten somit hinreichend Gelegenheit, den komplexen Mechanismus des Experiments zu durchschauen. Vgl. dazu auch Chow/Hwang/Liao (2000), S. 163.

${ }^{20}$ Im Vergleich dazu wurden den Teilnehmern bei Waller/Bishop (1990) nur drei Beispiele zur Funktionsweise des Groves-Mechanismus vorgelegt. Somit sollte das Verständnis für den GrovesMechanismus in dem hier vorliegenden Experiment besser sein, als dies bei Waller/Bishop der Fall war. Chow/Hwang/Liao (2000) bezeichnen zwar die ersten 15 von 20 Perioden ihres Experiments als Proberunden, den Teilnehmern war dies jedoch nicht bekannt, und die Proberunden wurden in gleicher Weise wie die Spielrunden entlohnt.

${ }^{21}$ Bei der Analyse der Kommunikation konnte die Experimentleitung weder einen Verstoß noch den Versuch eines Verstoßes gegen die Kommunikationsregeln feststellen.
} 
Zeitpunkt nicht mitgeteilt (ebenso wenig wie die Höhe dessen Entlohnung). Die tatsächliche Produktivität blieb somit während des gesamten Experiments private Information des jeweiligen Spielers. Dies wurde den Experimentteilnehmern in den Instruktionen mitgeteilt. Auch wurde ihnen erklärt, dass die zentrale Instanz nicht auf ihre tatsächliche Produktivität zurückschließen kann und sich damit Konsequenzen einer nicht wahrheitsgemäßen Berichterstattung nur über die Entlohnung ergeben. ${ }^{22}$

Um den Experimentteilnehmern Vergleiche zwischen den einzelnen Runden und unterschiedlichen Meldungen zu erleichtern, wurde ihnen sowohl nach jeder Probeals auch nach jeder Spielrunde eine Tabelle mit sämtlichen Werten der vorhergehenden Perioden ausgegeben.

Im Anschluss an das Experiment füllten alle Teilnehmer einen Fragebogen aus. Zwei der dort aufgeführten Fragen sollten das Verständnis der Funktionsweise des Groves-Mechanismus überprüfen. Den Teilnehmern des Treatments mit Kommunikation wurden darüber hinaus noch drei Fragen vorgelegt, die sich auf die Kommunikationsphase bezogen. Der Fragebogen ist ebenfalls dem Anhang zu entnehmen.

Das Experiment wurde mit 78 Studierenden und Angestellten der TU Clausthal am ExECUTe-Labor des Instituts für Wirtschaftswissenschaft der TU Clausthal durchgeführt. Am Treatment ohne Kommunikation nahmen 38 Spieler teil, am Treatment mit Kommunikation 40. Keine Sitzung dauerte länger als 135 Minuten. Alle Teilnehmer erhielten eine Prämie für ihr Erscheinen in Höhe von 10 EUR, die zusätzliche variable Vergütung aus den 10 Spielrunden betrug im Durchschnitt 12,92 EUR mit einem Minimum von 8,20 EUR und einem Maximum von 15,14 EUR. ${ }^{23}$

\section{Ergebnisse des Experiments}

Tabelle 2 gibt die deskriptive Statistik der im Experiment gewonnenen Daten wieder. Es zeigt sich, dass es weder im Treatment mit noch im Treatment ohne Kommunikation mehrheitlich zu wahrheitsgemäßer Berichterstattung kommt, was bereits gegen Hypothese 1a spricht. Während es im Fall ohne Kommunikation zumindest in 44,47\% der Fälle zu wahrheitsgemäßen Meldungen der Produktivitäten kommt, reduziert sich dies bei Kommunikation auf gerade einmal 21, 5\% der Fälle. Erstaunlicherweise liegen die Werte für das Treatment ohne Kommunikation relativ nahe an den Werten, die auch Waller/Bishop (1990) in ihrem Experiment zum GrovesMechanismus erhalten, obwohl hier zum einen die Teilnehmer im Vergleich zu den Teilnehmern von Waller/Bishop (1990) nicht nur drei Beispiele zur Entlohnung mit dem Groves-Mechanismus vorgelegt bekamen, sondern in 12 Proberunden dessen Funktionsweise selbst testen konnten, und sich zum anderen die verwendeten Gewinnfunktionen und Aufteilungsmechanismen unterscheiden. Dort wurden $48 \%$ der Meldungen wahrheitsgemäß abgegeben, 33\% der Meldungen waren Übertreibungen und 19\% Untertreibungen der Produktivität, während hier die Aufteilung für den Fall ohne Kommunikation $44,47 \%$ zu $36,58 \%$ zu 18, $95 \%$ beträgt. $^{24}$

\footnotetext{
${ }^{22}$ Theoretisch wäre für die zentrale Instanz in diesem Experimentrahmen ein solcher Rückschluss auf die tatsächliche Produktivität nach Realisation der tatsächlichen Gewinne der beiden Bereiche zwar möglich. Um jedoch Verzerrungen der Ergebnisse aufgrund unterschiedlicher Risikoeinstellungen, wie sie bei der Verwendung von Störtermen auftreten könnten, zu vermeiden, wurde dies ausgeschlossen.

${ }^{23}$ Das Experiment wurde mit Hilfe der Software „Toolkit for Economic Experiments with Communication" (TEEC) umgesetzt. Für weitere Informationen zu dieser Software: stefan.geisler@tuclausthal.de.

${ }^{24} \mathrm{Da}$, wie in Abschnitt 2 beschrieben, nicht jede Abweichung von der wahrheitsgemäßen Meldung auch automatisch zu einer Reduzierung der Entlohnung führt, war es durchaus möglich, dass sich auch für die Teilnehmer während der Proberunden bei geringen Abweichungen keine Auswirkungen auf die Entlohnung ergaben. Wertet man die Daten vor dem Hintergrund dieser Erfahrungen
} 
In Anlehnung an Waller/Bishop (1990) werden in der weiteren Analyse folgende Abweichungsmaße verwendet ${ }^{25}$ :

$$
\begin{aligned}
\Delta_{A} & =\widehat{p}_{i}-p_{i} \\
\Delta_{R} & = \begin{cases}\frac{\widehat{p}_{i}-p_{i}}{p_{i}-1,4} & \text { für } \widehat{p}_{i}<p_{i} \\
0 & \text { für } \widehat{p}_{i}=p_{i} \\
\frac{\widehat{p}_{i}-p_{i}}{2,1-p_{i}} & \text { für } \widehat{p}_{i}>p_{i}\end{cases}
\end{aligned}
$$

wobei $\widehat{p}_{i}$ die gemeldete Produktivität des Spielers $i$ und $p_{i}$ dessen tatsächliche Produktivität darstellen. Somit gibt $\Delta_{A}$ die absolute Abweichung von der tatsächlichen Produktivität, also die absolute Manipulation des Spielers, wieder und $\Delta_{R}$ die relative Manipulation, also die Abweichung von der wahrheitsgemäßen Berichterstattung bezogen auf das Manipulationspotential.

Der zweite und der dritte Teil von Tabelle 2 zeigen in Übereinstimmung mit der Tatsache, dass es mehr Über- als Untertreibungen gibt, dass die durchschnittlichen absoluten und relativen Abweichungen von der wahrheitsgemäßen Berichterstattung durchweg positiv sind, die Experimentteilnehmer also durchschnittlich höhere als ihre tatsächlichen Produktivitäten meldeten. Ein negativer Zeittrend, wie ihn Hypothese $1 \mathrm{~b}$ vorhersagt, scheint bereits hier fraglich. Für beide Treatments gilt, dass die durchschnittliche absolute und relative Manipulation in den Runden 610 höher sind als in den vorhergehenden Runden, obwohl der umgekehrte Fall zu erwarten gewesen wäre. Für den Fall ohne Kommunikation steigt die absolute Abweichung um $11 \%$, während die relative Abweichung jedoch annähernd konstant bleibt. Für das Treatment mit Kommunikation steigen dagegen sowohl die absolute als auch die relative Abweichung stark an: Während sich die absolute Manipulation um $21 \%$ erhöht, zeigt der dritte Teil der Tabelle, dass damit die Abweichung von der wahrheitsgemäßen Berichterstattung von durchschnittlich 46, 43\% auf 60,57\% des maximal möglichen Manipulationsbetrages zunimmt.

Mann-Whitney U-Tests der absoluten und relativen Abweichungen beider Treatments mit einer fiktiven Gruppe, die stets wahrheitsgemäß berichtet, weisen für beide Treatments höchst signifikante Unterschiede zur wahrheitsgemäßen Berichterstattung auf $(p<0,001)$. Hypothese 1a kann hier also bereits abgelehnt werden. Der Groves-Mechanismus führt im Experiment - im Gegensatz zur Vorhersage der Standardtheorie - nicht zu wahrheitsgemäßer Berichterstattung.

Tabelle 2 zeigt ebenfalls deutliche Unterschiede zwischen beiden Treatments auf, die sich theoretisch eben nicht unterscheiden dürften, handelt es sich ja bei der Kommunikation um "cheap talk", der sich nicht auf das Spiel auswirken dürfte. Jedoch wird in der Tabelle deutlich, dass es im Fall mit Kommunikation im Durchschnitt zu einer absoluten Manipulation der Produktivität in Höhe von 0,21475 kommt, während die durchschnittliche Abweichung ohne Kommunikation 0,0576, also nur gut $\frac{1}{4}$ davon beträgt. Ebenso deutlich unterscheiden sich die relativen Abweichungen beider Treatments. Mann-Whitney U-Tests zeigen auch hier für beide Abweichungsmaße höchst signifikante Unterschiede zwischen den beiden Gruppen $(p<0,001)$. Auch die Verteilung der Meldungen zwischen Untertreibung, wahrheitsgemäßer Meldung und Übertreibung verschiebt sich deutlich. Stehen für den Fall ohne Kommunikation zumindest noch 44,47\% wahrheitsgemäßer Meldungen

so aus, dass auch Meldungen als wahrheitsgemäße Berichte gewertet werden, die zwar nicht wahrheitsgemäß waren, deren Abweichung davon jedoch bei gegebener Meldung des Spielpartners nicht zu einer Änderung der Entlohnung führten, ergibt sich nun für den Fall ohne Kommunikation für die Aufteilung zwischen Wahrheit, Übertreibung und Untertreibung: 72,37\%, 20,53\%, 7, 10\%. Die Verteilung verschiebt sich also sehr stark zugunsten der wahrheitsgemäßen Berichterstattung. In der weiteren Analyse sollen jedoch nur die Originaldaten betrachtet werden.

${ }^{25}$ Vgl. auch Chow/Hwang/Liao (2000). 
36, 58\% Übertreibungen gegenüber, stellen im Treatment mit Kommunikation fast $\frac{3}{4}$ aller Meldungen Übertreibungen der Produktivität dar. Dies kann bereits als starkes Indiz für die Ablehnung von Hypothese 2 angesehen werden, es zeigt jedoch nicht, ob sich diese Unterschiede zwischen beiden Treatments aufgrund zufälliger Unterschiede in den Charakteristika der einzelnen Spieler ergeben oder ob sie aus Änderungen in den Strategien der Spieler folgen und somit direkt der Kommunikation zurechenbar wären. Darauf soll in Abschnitt 5 noch genauer eingegangen werden.

Abbildung 1 stellt die durchschnittlichen absoluten Abweichungen der beiden Treatments über die 10 Spielrunden dar. Wie aus der Abbildung deutlich zu erkennen ist, kann weder für das Treatment ohne noch für das Treatment mit Kommunikation ein Endspieleffekt festgestellt werden.

Eine Regressionsanalyse der relativen und absoluten Abweichungen ergibt für den Fall ohne Kommunikation keine signifikante Abhängigkeit von der Rundenzahl. Für den Fall mit Kommunikation dagegen zeigt sich eine stark signifikant positive Abhängigkeit (jeweils $p<0,01$ ).

Da die Kurve in Abbildung 1 für den Fall mit Kommunikation aber vor allem in den ersten 5 Perioden einen starken Aufwärtstrend aufweist und danach relativ konstant zu bleiben scheint, wurde die Regressionsanalyse noch einmal getrennt für die ersten und letzten 5 Runden durchgeführt. Ohne Kommunikation zeigt sich wiederum keine signifikante Abhängigkeit der relativen und absoluten Abweichung von der Rundenzahl. Dagegen ändern sich die Ergebnisse für das Treatment mit Kommunikation: Während die Abhängigkeit beider Abweichungen von der Rundenzahl in den ersten 5 Perioden weiterhin hoch signifikant ( $p<0,01$ in beiden Fällen) und positiv bleibt, wird der Regressionskoeffizient für die Runden 6-10 insignifikant. ${ }^{26}$

Diese Ergebnisse stehen im starken Widerspruch zu Hypothese 1b. In den letzten Runden kann generell kein negativer Zeittrend beobachtet werden, im Treatment mit Kommunikation in den ersten Runden dagegen sogar ein positiver. Hypothese 1b wird somit abgelehnt.

Eine mögliche Erklärung für die den Hypothesen 1a und 1b widersprechenden Ergebnisse besteht in der Verwendung des verhältnismäßig komplexen GrovesMechanismus. Dies hat zur Folge, dass es zum einen zu Problemen beim Verständnis des Mechanismus kommen kann: So zeigt Tabelle 3, die die Ergebnisse der Befragung der Experimentteilnehmer im Anschluss an die Durchführung des Experiments wiedergibt, dass von den beiden ersten Fragen, die das Verständnis des Groves-Mechanismus überprüften, Frage 1 von nur 56,41\% der Teilnehmer und Frage 2 von nur 57,69\% richtig beantwortet wurden. Zwar sind sich die Autoren der beschränkten Aussagekraft einer solchen Fragebogenauswertung bewusst, die Ergebnisse können jedoch zumindest als starker Hinweis auf Verständnisprobleme gewertet werden.

Zum anderen ist es im Groves-Mechanismus nicht möglich, aus der Meldung des Spielpartners eindeutig auf dessen Kooperationsbereitschaft zu schließen. Wie bereits oben erläutert, kann jeder Spieler aus der Meldung seines Partners immer nur einen probabilistischen Rückschluss auf dessen Kooperationsbereitschaft ziehen, indem er die Möglichkeit bewertet, dass dessen tatsächliche Produktivität kleiner ist als seine gemeldete. Dies macht im Umkehrschluss das Signalisieren der Kooperationsbereitschaft vergleichsweise teuer. Betrachtet man die Meldungen der Spieler paarweise, zeigen die Experimentdaten, dass es im Treatment ohne Kommunikation in 33 von 190 Fällen zu beidseitiger Übertreibung der Produktivitäten kam. Jedoch ist nur das Verhalten eines Paares eindeutig auf kollusives Verhalten zu Lasten

\footnotetext{
${ }^{26}$ Für die absolute Abweichung bleibt das Vorzeichen des Regressionskoeffizienten positiv, während es für die relative Abweichung negativ wird.
} 
der zentralen Instanz zurückzuführen. Die beidseitigen Übertreibungen der anderen Paare scheinen das Ergebnis individueller Strategien zu sein und treten nicht systematisch auf. ${ }^{27}$ In Übereinstimmung mit Waller/Bishop (1990), Chow/Hirst/Shields (1994) und Chow/Hwang/Liao (2000) zeigen sich also nur schwache Anzeichen für kollusives Verhalten der Experimentteilnehmer im Treatment ohne Kommunikation. Insgesamt deutet somit die durchschnittliche Übertreibung in diesem Treatment eher daraufhin, dass zum einen Verständnisprobleme, zum anderen soziale Präferenzen wie beispielsweise Altruismus die Meldung beeinflusst haben, als darauf, dass die Spieler versucht haben, Reputation als kooperativer Spieler aufzubauen.

Für den Fall mit Kommunikation ändern sich die theoretischen Vorhersagen zwar generell nicht, jedoch besteht nun für die Spieler im Fall, dass während der Kommunikationsphase eine Verabredung getroffen wird, die Möglichkeit, ein eindeutiges Signal bezüglich der Kooperationsbereitschaft des Spielpartners zu beobachten, nämlich die Einhaltung oder Verletzung der Absprache. Dies führte offensichtlich zu einem Kooperationsaufbau. Erstaunlich jedoch ist, dass es auch in den letzten Runden beinahe keinen Bruch der vereinbarten Kooperation zwischen den Spielern $\mathrm{gab}^{28}$ : Teil 2 von Tabelle 4 zeigt, dass selbst in der letzten Runde nur zwei Spieler von 32, bei denen im Zuge der Analyse der Kommunikation eine Absprache festgestellt werden konnte, von dieser abwichen. ${ }^{29}$ Eine potentielle Erklärung dafür ergibt sich aus der Analyse der Kommunikation. Sie zeigt, dass sich die Teilnehmer in den meisten Fällen gemeinschaftlich eine Meldestrategie erarbeiteten. Es fand somit offensichtlich in den ersten Perioden ein Vertrauensaufbau statt, der bewirkte, dass die Kooperation auch in den Folgeperioden nicht zusammenbrach. Der Anstieg der beiden Abweichungsmaße während der ersten Runden ist darauf zurückzuführen, dass immer mehr Spieler auf eine Strategie der gemeinschaftlichen Übertreibung übergingen. Die Kommunikation scheint also bedeutend mehr als nur "cheap talk" zu sein und führte hier sogar dazu, dass die Kooperation mehrheitlich über die gesamte Spieldauer aufrechterhalten werden konnte. Dies soll im folgenden Abschnitt genauer untersucht werden.

\section{Auswirkungen der Kommunikation}

In diesem Abschnitt wird einerseits die Kommunikation genauer analysiert, wobei insbesondere darauf eingegangen werden soll, wie oft und welche Verabredungen die Spieler trafen und ob diese auch eingehalten wurden. Andererseits soll auch untersucht werden, ob die Kommunikation die Meldestrategien veränderte. Bisher wurde nur gezeigt, dass sich die Meldungen im Treatment mit Kommunikation signifikant von denen im Treatment ohne Kommunikation unterscheiden, nicht jedoch, ob die Spieler dabei auch unterschiedliche Strategien verfolgten. Die höheren durchschnittlichen Abweichungen im Fall mit Kommunikation könnten schließlich auch auf die Tatsache zurückzuführen sein, dass im Vergleich zum Treatment ohne Kommunikation mehr Spieler der Meinung waren, dass Übertreibung die für sie beste Strategie darstellt. Tabelle 3 zeigt nämlich bei der Beantwortung der Frage 1 eine deutlich höhere Anzahl Spieler, die - gegeben die Meldung des Spielpartners - Übertreibung für die beste Strategie hielten.

\footnotetext{
${ }^{27}$ So ist zum einen mehrfach zu beobachten, dass bei hohen Produktivitäten übertrieben, bei niedrigen dagegen wahrheitsgemäß gemeldet wurde, und zum anderen, dass trotz niedriger Meldungen des Partners stets übertrieben wurde.

${ }^{28}$ Isaac/Walker (1988) finden ebenfalls in einem ihrer Treatments mit Kommunikation, dass sich eine stabile Kooperation auf sehr hohem Niveau einstellt. Allerdings saßen sich die Experimentteilnehmer in der Kommunikationsphase an einem Tisch gegenüber, konnten sich also identifizieren, was eine wesentlich stärkere Form der Kommunikation darstellt als der hier untersuchte anonyme Chat.

${ }^{29}$ Für eine genauere Analyse der Kommunikation sei auf den nächsten Abschnitt verwiesen.
} 
Zunächst soll jedoch die Kommunikation noch einmal genauer betrachtet werden. Die Möglichkeit zur Kommunikation und damit zur Absprache der Meldung wurde nur von 4 der 20 Paare nicht genutzt. Während ein Paar überhaupt nicht kommunizierte, konnten sich die 3 anderen Paare während der gesamten Spieldauer nicht einigen, wer von ihnen zuerst Informationen offen legen sollte. ${ }^{30}$ Die übrigen 32 Experimentteilnehmer konnten sich zumindest im Laufe des Experiments auf Absprachen verständigen. Tabelle 4 zeigt, dass sowohl die Mitteilungen der Produktivitäten (Zeile 4 von Teil 1) als auch die Anzahl der Absprachen (Zeile 3 von Teil 2) in den ersten Perioden tendenziell zunimmt. Dass Tabelle 4 nicht in jeder Periode 32 Miteilungen und Absprachen aufweist, hat verschiedene Ursachen: Zum einen haben sich nicht in jeder Runde alle 16 Paare abgesprochen. Zum anderen konnte bei der Analyse der Kommunikation nicht immer eindeutig eine beidseitige Absprache festgestellt werden. ${ }^{31}$ Dass die Anzahl der gegenseitigen Mitteilungen der Produktivitäten in jeder Runde kleiner ist als die Anzahl der Absprachen, liegt darin begründet, dass es teilweise sehr früh zu Absprachen über die Meldestrategien kam, die keiner weiteren Mitteilung der Produktivitäten bedurften (z.B. stets $2,1: 2,1$ melden).

Teil 1 von Tabelle 4 zeigt, dass die Produktivitäten dem Mitspieler in der Kommunikationsphase überwiegend wahrheitsgemäß mitgeteilt wurden, Über- und Untertreibungen dagegen nur eine untergeordnete Rolle spielen. Dies ist um so überraschender, als die Meldung der Produktivität an den Spielpartner der einzige Moment im Experiment ist, in dem der Spielpartner eine Information erhält, die er im Nachhinein nicht verifizieren kann. ${ }^{32}$ Geht man davon aus, dass nach einer Absprache übertriebene Produktivitäten an die Instanz gemeldet werden, kann für einen Spieler, der in dem Moment der Mitteilung an seinen Spielpartner untertreibt, die schließlich an die Instanz gemeldete Produktivität näher an seiner tatsächlichen (und damit der dominanten Strategie) liegen, als dies der Fall wäre, wenn er seinem Mitspieler seine tatsächliche Produktivität mitgeteilt hätte.

Der zweite Teil von Tabelle 4 zeigt, dass im Durchschnitt 95, 78\% aller Verabredungen eingehalten wurden. Im überwiegenden Maße bricht die Kooperation selbst in der letzten Runde nicht zusammen. Abweichungen von den Verabredungen sind zwar vereinzelt zu beobachten, die Analyse der Kommunikation zeigt jedoch, dass mehr oder weniger plausible Entschuldigungen ausreichten, um den Partner zu einer erneuten Kooperation zu bewegen. Wie bereits in der statistischen Analyse in Abschnitt 3 ist auch hier tatsächlich kein Endrundeneffekt festzustellen, was die Ablehnung von Hypothese 1b verstärkt. Im direkten Vergleich mit den vorhergehenden Runden stellt die letzte Runde noch nicht einmal die Runde mit der höchsten Anzahl Abweichungen von der vereinbarten Meldung dar. In Runde 7 kommt es sowohl absolut als auch prozentual, in Runde 1 zumindest prozentual gesehen zu mehr Abweichungen von den Absprachen. Ein Blick auf die letzte Frage in Tabelle 3 zeigt auch, dass sich immerhin 27 Spieler an die Absprache gebunden fühlten, obwohl de facto keinerlei vertragliche Bindung möglich war.

Im Zusammenhang mit den Abweichungen von den verabredeten Meldungen in frühen Runden wären auch Bestrafungen des jeweiligen Spielers vorstellbar. ${ }^{33}$ In

\footnotetext{
${ }^{30}$ Einer der Spieler bringt es während der Kommunikation auf die theoretisch richtige Lösung: "Ob eine Absprache was bringt - ich weiß es nicht! Ich kann dir ja nicht 100\% trauen, und das ist ja das Manko."

${ }^{31}$ In einigen Fällen wies ein Spieler eindeutig auf seine Meldung in der entsprechenden Runde hin, wohingegen die Antwort des Partners nicht eindeutig interpretiert werden konnte.

${ }^{32}$ Schwartz/Young (2002) untersuchen ebenfalls cheap talk in einem Budgetierungskontext und finden, dass die wahrheitsgemäße Mitteilung der Produktivität an den Mitspieler bei wiederholter Interaktion selbst ohne Möglichkeit der Verifikation im Nachhinein bei durchschnittlich $80 \%$ liegt. Vgl. Schwartz/Young (2002), S. 325-326.

${ }^{3}$ Die experimentelle Forschung zeigt, dass es trotz fehlender spieltheoretischer Gleichgewich-
} 
diesem Experiment wäre es beispielsweise möglich, seinen Mitspieler zu bestrafen, indem man eine niedrigere als die tatsächliche Produktivität meldet. Dies würde zwar unter Umständen auch eine Verringerung des eigenen tatsächlichen Gewinns mit sich bringen, da die Ressource nicht mehr optimal aufgeteilt wird, es würde jedoch auf jeden Fall auch den Mitspieler bestrafen.

Als Reaktion auf ein Abweichen von der Verabredung wäre es außerdem denkbar, den Partner dazu zu bringen, die maximale Produktivität an die Instanz zu melden, und selbst wahrheitsgemäß zu berichten. Auf diese Weise könnte der Mitspieler seinen zukünftigen Kooperationswillen signalisieren. Beide Strategien konnten jedoch nur jeweils ein Mal im Ansatz festgestellt werden, ein sicherer Rückschluss ist selbst in diesen Fällen aber nicht möglich.

Auch die Inhalte der Kommunikation sind weitgehend überraschend. Es wurden zwar hauptsächlich Produktivitäten ausgetauscht, die Spieler erklärten sich jedoch auch gegenseitig die Funktionsweise des Entlohnungsmechanismus, mögliche Manipulationsmöglichkeiten und sogar mögliche Konsequenzen, wenn statt der vereinbarten (übertriebenen) Produktivität die wahrheitsgemäße gemeldet würde. ${ }^{34}$ Offensichtlich waren sich die Spieler zumindest zum Teil tatsächlich des Gefangenendilemmas bewusst, in dem sie agierten. Darauf deutet auch hin, dass die Spieler sehr häufig betonten, dass sie "kooperative" Spieler seien bzw. kooperativ spielen würden, und sich bereits nach frühen Runden für die gelungene Kooperation bedankten.

Es bleibt nun noch zu analysieren, ob die Möglichkeit zur Kommunikation auch die Meldestrategien der Spieler beeinflusst hat und somit auch Hypothese 2 abgelehnt werden muss. Obwohl, wie bereits gezeigt, sich die Meldungen in beiden Treatments signifikant voneinander unterschieden, kann dies auch einfach darauf beruhen, dass sich der Anteil Spieler verringert, der c.p. Untertreibung für die beste Strategie hält, bzw. der Anteil Spieler erhöht, der c.p. Übertreibung als beste Strategie erachtet. Diese Verschiebung zeigt sich in Frage 1 von Tabelle 3. Erst ein signifikanter Unterschied in den Strategien der Spieler könnte die signifikanten Unterschiede in den Meldungen tatsächlich auf die Möglichkeit der Kommunikation zurückführen.

In Tabelle 5 sind deshalb die durchschnittlichen absoluten und relativen Abweichungen in Abhängigkeit der Beantwortung von Frage 1 des Fragebogens aufgeführt. Die erste Spalte zeigt, dass für den Fall ohne Kommunikation sowohl die absolute als auch die relative Manipulation stark abnehmen, wenn man von Übertreibung zu wahrheitsgemäßer Berichterstattung übergeht, und schließlich sogar negativ wird, wenn auf Untertreibung übergegangen wird. Mann-Whitney U-Tests zeigen sowohl für $\Delta_{A}$ als auch für $\Delta_{R}$, dass diese Unterschiede zwischen den Gruppen hoch signifikant sind $(p<0,01) .{ }^{35}$ Obwohl zwar im Durchschnitt Übertreibungen zu beobachten sind, unterscheiden sich die drei Gruppen jedoch signifikant hinsichtlich ihrer gemeldeten Produktivitäten, und die Höhe der Abweichungen tendiert in die Richtung, die auch die Beantwortung der Frage impliziert.

Im Treatment mit Kommunikation sinken die durchschnittlichen absoluten und relativen Abweichungen ebenfalls in Abhängigkeit der Beantwortung von Frage 1 des Fragebogens, jedoch bei weitem nicht mehr so stark wie im Fall ohne Kommunikation. Mann-Whitney U-Tests für beide Abweichungsmaße zeigen nun bei den

te durchaus zu Bestrafungen unkooperativer Teammitglieder kommen kann. Vgl. Fehr/Gächter (2000), Sefton/Shupp/Walker (2001), Carpenter (2002).

${ }^{34}$ Nach Absprache auf eine Meldestrategie $(2,1: 2,1)$ bringt es ein Spieler während der Kommunikationsphase folgendermaßen auf den Punkt: "Die Sache ist die: Wenn einer weniger bietet, dann ist er zwar im Vorteil, aber sein Mitspieler, naja, der heult rum, und das ist ja nicht so schön, wenn wir hier anfangen, uns zu bekriegen."

${ }^{35}$ Alle bis auf einen Test weisen sogar ein Signifikanzniveau $p<0,001$ auf. 
paarweisen Vergleichen zwischen allen drei Gruppen keine signifikanten Unterschiede mehr ( $p>0,1$ in allen Fällen). Die Einschätzung, welche Meldung c.p. die beste ist, hat nun keinen signifikanten Einfluss mehr auf das tatsächliche Verhalten.

Offensichtlich verändern sich also die Meldestrategien: Im Vergleich zu den Teilnehmern im Fall ohne Kommunikation übertrieben die Spieler im Treatment mit Kommunikation nun ihre Meldungen unabhängig davon, was sie c.p. für sich selbst als beste Meldung erachteten. Tendieren die Meldungen der Spieler im Treatment ohne Kommunikation noch in die Richtung, die sie c.p. für sich selbst als optimal ansahen, meldeten sie stattdessen im Treatment mit Kommunikation Produktivitäten, die für beide Spielpartner zusammen von Vorteil waren. Hypothese 2 ist also endgültig abzulehnen.

Die Analyse dieses Abschnittes hat gezeigt, dass die Kommunikation erheblichen Einfluss auf das Verhalten der Experimentteilnehmer hat, obwohl sich die theoretischen Vorhersagen für beide Treatments nicht unterscheiden. In Übereinstimmung mit der bisherigen Literatur zu Kommunikationsexperimenten führt die Kommunikation dazu, dass sich die Experimentteilnehmer wesentlich kooperativer verhalten. In den ersten 5 Runden nimmt die Kooperation stark zu, und es entwickeln sich stabile Kooperationsstrategien, die dazu führen, dass nicht einmal in den letzten Runden ein Zusammenbrechen der Kooperation zu beobachten ist. Dieses kooperative Verhalten machte sich für die Teilnehmer auch bezahlt: Im Durchschnitt erhielten die Teilnehmer im Treatment mit Kommunikation eine variable Entlohnung aus dem Experiment in Höhe von 13, 35 EUR, im Fall ohne Kommunikation dagegen von nur 12, 48 EUR.

\section{Zusammenfassung}

Der Groves-Mechanismus als Anreizsystem zur wahrheitsgemäßen Berichterstattung wird gerade im deutschsprachigen Raum in den letzten Jahren stark diskutiert. Insbesondere steht dabei die Frage im Mittelpunkt, warum dieser Mechanismus nicht in der Unternehmenspraxis zu beobachten ist. Mehrere Beiträge stellen seine Optimalität bei zusätzlich existierenden moral hazard-Problemen in Frage. Alleine jedoch die Frage, ob der Groves-Mechanismus ohne diese zusätzliche Problematik eine wahrheitsinduzierende Wirkung hat, ist zwar theoretisch abschließend geklärt, wurde bisher jedoch nur ansatzweise im Experiment untersucht. Dieser Beitrag ergänzt und erweitert die bisherige experimentelle Forschung auf diesem Gebiet. Durch die Berücksichtigung von Kommunikation wird zudem ein stärkerer Bezug auf die Gegebenheiten in der Praxis hergestellt.

Wie relevant aber eine experimentelle Überprüfung der theoretischen Ansätze ist, zeigt sich daran, dass alle drei auf Basis der theoretischen Überlegungen hergeleiteten Hypothesen abgelehnt werden müssen. Die Ergebnisse lassen erkennen, dass es beim Groves-Mechanismus bereits ohne Kommunikation mehrheitlich nicht zu wahrheitsgemäßer Berichterstattung kommt. Können die Spieler vor Abgabe ihrer Meldung miteinander kommunizieren, verringert sich der Anteil wahrheitsgemäßer Berichterstattung weiter.

Trotz dieser verzerrten Berichterstattung zeigen sich in Übereinstimmung mit früheren Experimenten aber auch hier nur schwache Anzeichen für ein gemeinschaftlich kollusives Verhalten zur Lasten der Instanz, wenn die Spieler keine Möglichkeit zur Kommunikation besitzen. Dies ändert sich allerdings maßgeblich im Treatment mit Kommunikation. Obwohl diese vollkommen anonym ist und wohl eine der schwächsten Formen der Kommunikation darstellt, kommt es nun zu stabilen Manipulations- und Kooperationsstrategien der Experimentteilnehmer. Auf- 
grund dieser Ergebnisse der beiden Treatments erstaunt es nicht, dass der GrovesMechanismus trotz seiner wünschenswerten theoretischen Eigenschaften in der Praxis nicht vorzufinden ist.

Neben diesen Ergebnissen liefert der Beitrag zusätzliche Erkenntnisse über die Auswirkungen von Kommunikationsmöglichkeiten auf wirtschaftswissenschaftlich relevante Fragestellungen und im Besonderen auf die Fragestellung nach Kooperation in endlich wiederholten Gefangenendilemmata. Dabei führt der hier verwendete komplexe Groves-Mechanismus im Vergleich zu den relativ einfachen Spielstrukturen anderer Experimente in diesem Bereich zu einer noch stärkeren Veränderung des kooperativen Verhaltens bei Kommunikation.

Die Ergebnisse des vorgestellten Experiments zeigen also, dass Kommunikationsmöglichkeiten im Gegensatz zu den Vorhersagen der Standardtheorie erhebliche Auswirkungen auf betriebswirtschaftliche Entscheidungen haben können. Weitere Untersuchungen in diesem Gebiet könnten deshalb Erklärungsbeiträge zu empirischen Regelmäßigkeiten liefern, die unter standardtheoretischen Annahmen nicht erklärbar sind, zumal in Unternehmen Kommunikation in vielfältiger Art und Weise möglich ist. Eine Vernachlässigung der Kommunikation als "cheap talk" scheint dagegen nicht angebracht. 


\section{Anhang: Instruktionen zum Experiment}

Sie sind Teilnehmer an einem Experiment zum individuellen Entscheidungsverhalten. Während des Experiments werden Sie eine Reihe von Entscheidungen zu treffen haben, die Ihre Entlohnung am Ende des Experiments beeinflussen.

Der Gesamtbetrag, den Sie während des Experiments erzielen, wird Ihnen in der nächsten Woche ausbezahlt.

Sowohl Ihre Entscheidungen als auch Ihre Entlohnung sind nur Ihnen bekannt, den anderen Experimentteilnehmern werden sie nicht mitgeteilt. Auch die Experimentleitung kann Ihre Entscheidungen nicht mit Ihrer Person identifizieren.

Am Ende der Erläuterungen zu diesem Experiment haben Sie noch die Möglichkeit, Fragen zu stellen, falls Ihnen etwas unklar geblieben ist.

Das Experiment besteht aus 10 Runden. Dabei werden Sie in allen 10 Runden mit dem gleichen Partner spielen, dessen Identität Ihnen aber nicht bekannt ist.

Zu Beginn jeder Runde bekommen Sie eine Produktivität mitgeteilt. Diese Produktivität sagt Ihnen, wie gut Sie die Einheiten einer Ressource einsetzen können, um einen Gewinn zu erwirtschaften. Auf die genaue Höhe des Gewinns wird später eingegangen.

Auf die Höhe der Produktivität haben Sie keinen Einfluss. Sie wird in jeder Periode per Zufall neu bestimmt und Ihnen mitgeteilt.

Von der Ressource, die zwischen Ihnen und Ihrem Spielpartner aufgeteilt wird, sind maximal 120 Einheiten verfügbar. Diese werden von einer zentralen Instanz verwaltet, an die Sie und Ihr Spielpartner gleichzeitig jeweils eine Produktivität berichten müssen. Welche Produktivität Ihr Spielpartner berichtet, wissen Sie bei Abgabe Ihrer Meldung nicht.

Die Produktivität, die Sie an die zentrale Instanz melden, kann, muss aber nicht mit Ihrer tatsächlichen Produktivität übereinstimmen. Nur Sie kennen dabei Ihre genaue Produktivität $(\mathrm{P})$ in jeder Runde. Ihr Spielpartner und die zentrale Instanz erfahren diese nicht und können diese auch nicht rechnerisch aus den bekannt gewordenen Daten ermitteln.

Ihre Produktivität (P) kann in Schritten von 0,1 zwischen 1,4 und 2,1 liegen und wird zu Beginn jeder Runde zufällig neu bestimmt und Ihnen dann mitgeteilt.

Sie können damit folgenden Gewinn erzielen:

i) Werden Ihnen 40 Ressourceneinheiten (RE) oder weniger zugeteilt, beträgt der Gewinn, den Sie erzielen können:

Gewinn $=\mathrm{P}$. Anzahl RE - Anzahl RE $=(\mathrm{P}-1) \cdot$ Anzahl RE

Alle zugeteilten Ressourceneinheiten können in diesem Fall also zu der tatsächlichen Produktivität angelegt werden, die Ihnen zu Beginn der Runde von dem Programm mitgeteilt wird.

ii) Werden Ihnen zwischen 40 und 100 Einheiten zugeteilt, können Sie die Einheiten, die über 40 hinausgehen, jedoch nicht mehr zu der Produktivität anlegen, die Ihnen zu Beginn der Runde mitgeteilt wurde, sondern nur noch zu einer Produktivität, die um 0,3 geringer ist. Werden Ihnen also mehr als 40, aber höchstens 100 RE zugeteilt, beträgt Ihr Gewinn:

Gewinn $=40 \cdot \mathrm{P}+($ Anzahl RE -40$) \cdot(\mathrm{P}-0,3)-$ Anzahl RE

iii) Zusätzliche Einheiten der Ressource, die über 100 hinausgehen, können Sie nicht mehr produktiv einsetzen.

Nachdem Sie zu Beginn jeder Runde Ihre Produktivität mitgeteilt bekommen haben, müssen Sie gleichzeitig mit Ihrem Spielpartner jeweils eine Produktivität an die zentrale Instanz melden.

Ihre gemeldete Produktivität muss dabei nicht mit Ihrer tatsächlichen Produktivität übereinstimmen. Es sei nochmals daran erinnert, dass während des gesamten 
Experiments nur Sie Ihre tatsächliche Produktivität jeder Runde kennen und sonst niemand.

Die zentrale Instanz wird nach Ihrer Meldung die verfügbaren 120 Ressourceneinheiten (RE) auf Sie und Ihren Spielpartner aufteilen. Die Aufteilung erfolgt auf Basis Ihrer Meldung und der Meldung Ihres Spielpartners. Dabei gilt:

i) Berichten Sie eine Produktivität, die genauso hoch ist wie die Ihres Spielpartners, bekommen Sie beide 60 RE zugeteilt.

ii) Melden Sie eine Produktivität, die größer ist als die gemeldete Produktivität Ihres Spielpartners, aber nicht größer als die Produktivität Ihres Spielpartners plus 0,3, bekommen Sie 80 RE, Ihr Partner 40 RE.

iii) Berichten Sie eine Produktivität, die um mehr als 0,3 höher ist als die Ihres Spielpartners, erhalten Sie 100 Einheiten, Ihr Partner 20.

Das Umgekehrte gilt, wenn Ihre gemeldeten Produktivitäten kleiner sind als die Ihres Spielpartners.

In jeder Runde erhalten Sie Punkte, die die Basis für Ihre Entlohnung am Ende des Experiments bilden. Beachten Sie, dass 7 Punkte am Ende 1 EUR wert sein werden. Teilen Sie also die Anzahl der Punkte durch 7, erhalten Sie als Ergebnis Ihre Entlohnung. Punkte pro Runde $=0,1 \cdot$ (tatsächlicher eigener Gewinn + gemeldeter Gewinn des Spielpartners)

In jeder Runde erhalten Sie demnach 10\% der Summe aus Ihrem eigenen tatsächlichen Gewinn und dem gemeldeten Gewinn Ihres Spielpartners als Punkte.

Ihr eigener tatsächlicher Gewinn berechnet sich dabei auf Basis Ihrer tatsächlichen Produktivität und der Ihnen zugeteilten RE, wie es oben erläutert wurde. Er beträgt somit je nach Anzahl der zugeteilten RE:

i) Für $\mathrm{RE}=20$ oder $\mathrm{RE}=40$ :

Gewinn $=\mathrm{P}$. Anzahl RE - Anzahl RE $=(\mathrm{P}-1) \cdot$ Anzahl RE

ii) $\mathrm{Für} \mathrm{RE}=60, \mathrm{RE}=80$ oder $\mathrm{RE}=100$ :

Gewinn $=40 \cdot \mathrm{P}+($ Anzahl RE -40$) \cdot(\mathrm{P}-0,3)-$ Anzahl RE

Der gemeldete Gewinn Ihres Spielpartners muss nicht seinem tatsächlichen Gewinn entsprechen. Der gemeldete Gewinn Ihres Spielpartners berechnet sich auf Basis der Produktivität, die er an die Zentrale meldet, und der RE, die ihm zugeteilt werden. Wie hoch der tatsächliche Gewinn Ihres Spielpartners ist, ist für Ihre Entlohnung nicht relevant.

Ihre Entlohnung am Ende des Experiments ist die Summe der einzelnen Punkte, die Sie in den 10 Spielrunden erhalten haben, geteilt durch 7.

Eine Zusammenfassung der Formeln wird Ihnen jetzt ausgeteilt.

Um Erfahrungen mit diesem Entlohnungsmechanismus zu sammeln, können Sie 12 Proberunden spielen, in denen jeweils die gemeldeten Produktivitäten Ihres Spielpartners simuliert werden und Ihnen zu Beginn mitgeteilt werden. Das bedeutet, dass Sie während der Proberunden keinen tatsächlichen Spielpartner haben. Stattdessen werden Ihnen mögliche Meldungen eines Spielpartners fest vorgegeben.

Ihr Spielpartner wird Ihnen erst nach den Proberunden zufällig zugeteilt.

Während der 10 Spielrunden werden Sie Ihre Entscheidung zu treffen haben, ohne dass Sie die Meldung Ihres Spielpartners kennen.

- In den Proberunden 1-6 beträgt die gemeldete Produktivität des Spielpartners 1,5 .

- In den Proberunden 7-12 beträgt die gemeldete Produktivität des Spielpartners 1,9 . 
Auch Ihre eigene, tatsächliche Produktivität wird Ihnen für diese 12 Proberunden fest vorgegeben.

- In den Proberunden 1-3 beträgt Ihre tatsächliche Produktivität 1,5.

- In den Proberunden 4-6 beträgt Ihre tatsächliche Produktivität 2,0.

- In den Proberunden 7-9 beträgt Ihre tatsächliche Produktivität 1,5.

- In den Proberunden 10-12 beträgt Ihre tatsächliche Produktivität 2,0.

$\mathrm{Zu}$ Beginn jeder Proberunde erscheinen sowohl Ihre eigene tatsächliche Produktivität als auch die gemeldete Produktivität des Spielpartners, die für die jeweilige Runde angenommen wird, auf Ihrem Bildschirm.

Die Proberunden haben keinen Einfluss auf Ihre Entlohnung, die Sie am Ende des Experiments erhalten.

Nur die Ergebnisse der 10 Spielrunden, die im Anschluss an die Proberunden stattfinden, und die Punkte, die Sie während dieser Spielrunden sammeln, ergeben wie vorher beschrieben Ihre Gesamtentlohnung für dieses Experiment.

Während des gesamten Experiments darf nicht gesprochen werden.

Treffen Sie Ihre Entscheidung für sich alleine, ohne Kontakt zu anderen Experimentteilnehmern zu suchen.

Ihr Spielpartner wird für Sie anonym bleiben.

Beachten Sie, dass Sie Ihre Entlohnung streng vertraulich erhalten. Weder die Experimentleitung noch andere Experimentteilnehmer können Sie anhand Ihrer Eingaben identifizieren.

Falls Sie sich etwas notieren möchten, können Sie das gerne tun, Sie müssen die Zettel am Ende des Experiments jedoch abgeben.

\section{Zusatz für das Treatment mit Kommunikation:}

Dabei wird Ihnen zu Beginn jeder Runde Ihre tatsächliche Produktivität für die entsprechende Runde mitgeteilt. Bevor Sie Ihre Meldung abgeben, haben Sie die Gelegenheit, mit Ihrem Spielpartner zu kommunizieren.

Dazu wird sich für Sie ein Chatfenster öffnen, in dem Sie Ihre Nachrichten Ihrem Spielpartner mitteilen können und Ihrerseits seine Nachrichten lesen können.

Für diese Kommunikation haben Sie je nach Runde unterschiedlich lange Zeit. Mit zunehmender Rundenanzahl wird dabei die Dauer der Kommunikation eingeschränkt. Wie lange Sie Zeit haben, wird Ihnen zu Beginn jeder Kommunikationsphase mitgeteilt.

\section{Regeln für die Kommunikation:}

Für die Kommunikation gelten folgende Regeln:

i) Sie dürfen sich nicht identifizieren. Nennen Sie weder Ihren Namen noch Ihren Studiengang noch sonstige Details über sich.

ii) Es ist Ihnen nicht erlaubt, Ihren Spielpartner zu beschimpfen oder ihm mit etwas zu drohen, das über das Spiel hinausgeht. Auch andere Verabredungen über das Spiel hinaus sind nicht erlaubt.

iii) Sie dürfen nur auf Deutsch kommunizieren. Die Verwendung von Fremdsprachen ist nicht erlaubt.

Die Experimentleitung wird Ihre Kommunikation nach dem Experiment auf diese Regeln hin überprüfen. Stellt sich heraus, dass Sie gegen eine oder mehrere Regeln verstoßen haben, erhalten Sie außer den 10 EUR für Ihr Erscheinen keine Entlohnung!

Bitte warten Sie, bis Ihnen diese Regeln zur Kommunikation noch einmal auf einem Zettel ausgeteilt werden. 


\section{Fragebogen am Ende des Experiments:}

Die Beantwortung dieses Fragebogens wird keinen Einfluss auf die Höhe Ihrer Entlohnung nehmen. Trotzdem möchten wir Sie darum bitten, die unten aufgeführten Fragen sorgfältig zu lesen und zu beantworten.

\section{Frage 1:}

Wenn Sie nur ein einziges Mal mit Ihrem Spielpartner zusammen spielen würden und bereits wüssten, wie er sich entschieden hat, wäre es für Sie vorteilhaft,

- eine höhere als Ihre tatsächliche Produktivität zu melden.

- Ihre tatsächliche Produktivität zu melden.

- eine niedrigere als Ihre tatsächliche Produktivität zu melden.

Frage 2:

Wenn Sie Ihre Entscheidung bereits getroffen hätten und nicht mehr ändern könnten, wäre es für Sie vorteilhaft, wenn Ihr Spielpartner

- eine höhere als seine tatsächliche Produktivität meldet.

- seine tatsächliche Produktivität meldet.

- eine niedrigere als seine tatsächliche Produktivität meldet.

Frage 3: (nur beim Treatment mit Kommunikation)

Hat die Möglichkeit zur Absprache mit Ihrem Mitspieler Ihre Meldung beeinflusst?

- ja

- nein

Frage 4: (nur beim Treatment mit Kommunikation)

Für den Fall, dass Ihnen Ihr Spielpartner seine Produktivität während der Kommunikationsphase mitgeteilt hat: Sind Sie davon ausgegangen, dass diese Produktivität seiner tatsächlichen Produktivität entsprach?

- ja

- nein

- Es wurde keine Produktivität mitgeteilt.

Frage 5: (nur beim Treatment mit Kommunikation)

Für den Fall, dass Sie sich in der letzten Runde abgesprochen haben: Fühlten Sie sich durch die Absprache gebunden?

- ja

- nein

- Es wurde keine Absprache getroffen. 


\section{Literatur}

Andreoni, James/Miller, John H. (1993): Rational Cooperation in the Finitely Repeated Prisoner's Dilemma: Experimental Evidence, in: The Economic Journal, 103, 570-585.

Arbeitskreis „Finanzierung“ der Schmalenbach-Gesellschaft für Betriebswirtschaft e.V. (1994): Investitions-Controlling - Zum Problem der Informationsverzerrung bei Investitionsentscheidungen in dezentralisierten Unternehmen, in: Zeitschrift für betriebswirtschaftliche Forschung, 46, 899-925.

Bamberg, Günter/Locarek, Hermann (1992): Groves-Schemata zur Lösung von Anreizproblemen bei der Budgetierung, in: Spremann, Klaus/Zur, Eberhard (Hrsg.): Controlling: Grundlagen - Informationssysteme - Anwendungen, 657-670.

Banker, Rajiv D./Datar, Srikant M. (1992): Optimal Transfer Pricing under Postcontract Information, in: Contemporary Accounting Research, 8, 329-352.

Bolton, Gary/Chatterjee, Kalyan/McGinn, Kathleen L. (2003): How Communication Links Influence Coalition Bargaining: A Laboratory Investition, in: Management Science, 49, 583-598.

Brosig, Jeannette/Weimann, Joachim/Ockenfels, Axel (2003): The Effect of Communication Media on Cooperation, in: German Economic Review, 4, 217-241.

Budde, Jörg/Göx, Robert F./Luhmer, Alfred (1998): Absprachen beim GrovesMechanismus - eine spieltheoretische Untersuchung, in: Zeitschrift für betriebswirtschaftliche Forschung, 50, 3-20.

Camerer, Colin/Weigelt, Keith (1988): Experimental Tests of a Sequential Equilibrium Reputation Model, in: Econometrica, 56, 1-36.

Carpenter, Jeffrey P. (2002): Punishing Free-Riders: How Group Size Affects Mutual Monitoring and the Provision of Public Goods, Discussion Paper, Middlebury College.

Chow, Chee W./Hirst, Mark/Shields, Michael D. (1994): Motivating Truthful Subordinate Reporting: An Experimental Investigation in Two-Subordinate Context, in: Contemporary Accounting Research, 10, 699-720.

Chow, Chee W./Hirst, Mark/Shields, Michael D. (1995): The Effects of Pay Schemes and Probabilistic Management Audits on Subordinate Misrepresentation of Private Information: An Experimental Investigation in a Resource Allocation Context, in: Behavioral Research in Accounting, 7, 1-16.

Chow, Chee W./Hwang, Richard Nen-Chen/Liao, Woody (2000): Motivating Truthful Upward Communication of Private Information: An Experimental Study of Mechanisms from Theory and Practice, in: Abacus, 36, 160-179.

Clarke, Edward H. (1971): Multipart Pricing of Public Goods, in: Public Choice, 8, 17-33.

Cohen, Susan I./Loeb, Martin (1984): The Groves Scheme, Profit Sharing and Moral Hazard, in: Management Science, 30, 20-24.

Cooper, Russel/DeJong, Douglas V./Forsythe, Robert/Ross, Thomas W. (1996): Cooperation without Reputation: Experimental Evidence from Prisoner's Dilemma Games, in: Games and Economic Behavior, 12, 187-218.

Davis, Douglas D./Holt, Charles A. (1993): Experimental Economics, Princeton Press.

Ewert, Ralf/Wagenhofer, Alfred (2000): Interne Unternehmensrechnung, 4. Auflage, Berlin et al. 
Fehr, Ernst/Gächter, Simon (2000): Cooperation and Punishment in Public Goods Experiments, in: American Economic Review, 90, 980-994.

Groves, Theodore (1973): Incentives in Teams, in: Econometrica, 41, 617-631.

Groves, Theodore/Loeb, Martin (1979): Incentives in a Divisionalized Firm, in: Management Science, 25, 221-230.

Hannan, R. Lynn/Rankin, Frederick W./Towry, Kristy L. (2003): Budgeting Without Commitment: The Effect of Non-Contractible Information on Honesty in Managerial Reporting, Working Paper, Georgia State University.

Hofmann, Christian/Pfeiffer, Thomas (2003) Investitionsbudgetierung und Anreizprobleme: Ist der Groves-Mechanismus nur third-best? Zur Effizienz des GrovesBudgetierungsmechanismus, in: Zeitschrift für Betriebswirtschaft, 73, 559-582.

Isaac, R. Mark/McCue, Kenneth F./Plott, Charles R. (1985): Public Goods Provision in an Experimental Environment, in: Journal of Public Economics, 26, 51-74.

Isaac, R. Mark/Walker, James (1988): Communication and Free Riding Behavior: The Voluntary Contribution Mechanism, in: Economic Inquiry, 26, 585-608.

Jensen, Michael C. (2001a): Paying People to Lie: The Truth About the Budgeting Process, Working Paper, Harvard Business School.

Jensen, Michael C. (2001b): Corporate Budgeting Is Broken - Let's Fix It, in: Harvard Business Review, November 2001, 94-101.

Krapp, Michael (2000): Relative Leistungsbewertung im dynamischen Kontext Eine Analyse der Kollusionsionsproblematik bei wiederholter Delegation, in: Zeitschrift für betriebswirtschaftliche Forschung, 52, 257-277.

Kreps, David M./Milgrom, Paul/Roberts, John/Wilson, Robert (1982): Rational Cooperation in the Finitely Repeated Prisoners' Dilemma, in: Journal of Economic Theory, 27, 245-252.

Kunz, Alexis H./Pfeiffer, Thomas (1999): Investitionsbudgetierung und implizite Verträge: Wie resistent ist der Groves-Mechanismus bei dynamischer Interaktion?, in: Zeitschrift für betriebswirtschaftliche Forschung, 51, 203-223.

Laux, Helmut (1999): Unternehmensrechnung, Anreiz und Kontrolle, 2. Auflage, Berlin et. al.

Laux, Helmut/Liermann, Felix (2003): Grundlagen der Organisation, 5. Auflage, Berlin et al.

Ledyard, John O. (1995): Public Goods: A Survey of Experimental Research, in: Kagel, John H./Roth, Alvin E. (Hrsg.): Handbook of Experimental Economics, Princeton Press, 111-194.

Locarek, Hermann/Bamberg, Günter (1994): Anreizkompatible Allokationsmechanismen für divisionalisierte Unternehmungen, in: Wirtschaftswissenschaftliches Studium, 23, 10-14.

Osband, Kent/Reichelstein, Stefan J. (1985): Information-Eliciting Compensation Schemes, in: Journal of Public Economics, 27, 107-115.

Pfaff, Dieter/Leuz, Christian (1995): Groves-Schemata - Ein geeignetes Instrument zur Steuerung der Ressourcenallokation in Unternehmen?, in: Zeitschrift für betriebswirtschaftliche Forschung, 47, 659-690.

Reichelstein, Stefan J./Osband, Kent (1984): Incentives in Government Contracts, in: Journal of Public Economics, 24, 257-270. 
Schwartz, Steven T./Young, Richard A. (2002): A Laboratory Investigation of Verification and Reputation Formation in a Repeated Joint Investment Setting, in: Contemporary Accounting Research, 19, 311-342.

Sefton, Martin/Shupp, Robert/Walker, James (2001): The Effect of Rewards and Sanctions in Provision of Public Goods, Working Paper, Indiana University.

Vickrey, William (1964): Counterspeculation, Auctions, and Competitive Sealed Tenders, in: The Journal of Finance, 16, 8-37.

Waller, William S./Bishop, Rachel A. (1990): An Experimental Study of Incentive Pay Schemes, Communication, and Intrafirm Resource Allocation, in: The Accounting Review, 65, 812-836.

Weitzman, Martin L. (1976): The New Soviet Incentive Model, in: Bell Journal of Economics, 7, 251-257. 


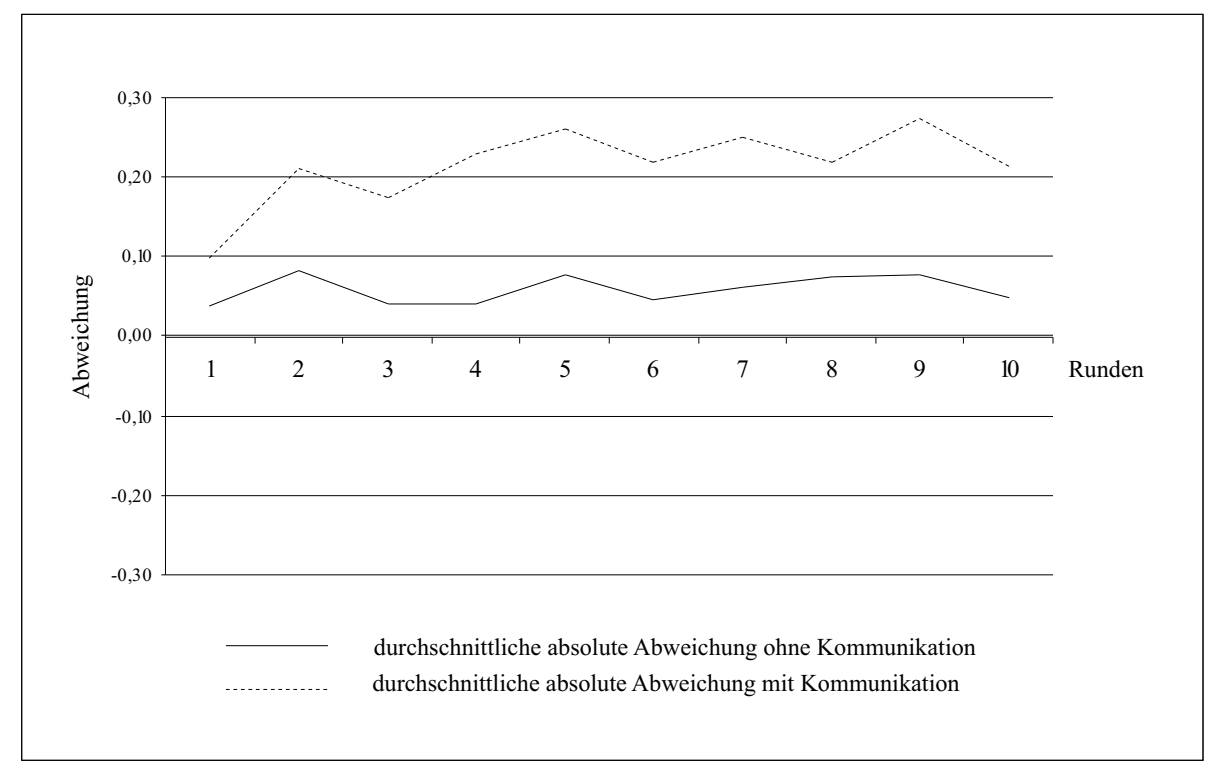

Abbildung 1: Durchschnittliche absolute Abweichung von der wahrheitsgemäßen Meldung der Produktivität.

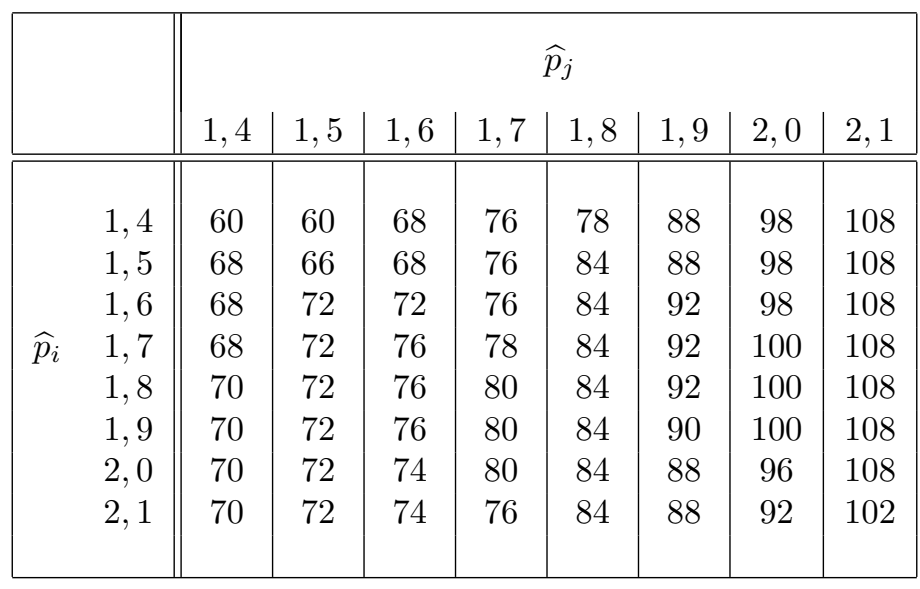

Tabelle 1: Bemessungsgrundlage für Manager i bei einer tatsächlichen Produktivität seines Bereiches von 1,8. 


\begin{tabular}{|c|c|c|c|}
\hline $\begin{array}{l}\text { Teil } 1 \\
\text { Anzahl der Meldungen }\end{array}$ & $\begin{array}{c}\text { ohne } \\
\text { Kommunikation }\end{array}$ & $\begin{array}{c}\text { mit } \\
\text { Kommunikation }\end{array}$ & Gesamt \\
\hline Untertreibung & $\begin{array}{c}72 \\
(18,95 \%)\end{array}$ & $\begin{array}{c}23 \\
(5,75 \%)\end{array}$ & $\begin{array}{c}95 \\
(12,18 \%)\end{array}$ \\
\hline Wahrheitsgemäße Meldung & $\begin{array}{c}169 \\
(44,47 \%)\end{array}$ & $\begin{array}{c}86 \\
(21,50 \%)\end{array}$ & $\begin{array}{c}255 \\
(32,69 \%)\end{array}$ \\
\hline Übertreibung & $\begin{array}{c}139 \\
(36,58 \%)\end{array}$ & $\begin{array}{c}291 \\
(72,75 \%)\end{array}$ & $\begin{array}{c}430 \\
(55,13 \%)\end{array}$ \\
\hline & $\begin{array}{c}380 \\
(100 \%)\end{array}$ & $\begin{array}{c}400 \\
(100 \%) \\
\end{array}$ & $\begin{array}{c}780 \\
(100 \%) \\
\end{array}$ \\
\hline \multicolumn{4}{|c|}{$\begin{array}{l}\text { Teil } 2 \\
\text { Durchschnittliche absolute Abweichung }\left(\Delta_{A}\right)\end{array}$} \\
\hline Runden 1-5 & 0,0547 & 0,1945 & 0,1264 \\
\hline Runden 6-10 & 0,0605 & 0,2350 & 0,1500 \\
\hline Gesamt & 0,0576 & 0,21475 & 0,1382 \\
\hline \multicolumn{4}{|c|}{$\begin{array}{l}\text { Teil } 3 \\
\text { Durchschnittliche relative Abweichung }\left(\Delta_{R}\right)\end{array}$} \\
\hline Runden 1-5 & 0,1604 & 0,4643 & 0,3162 \\
\hline Runden 6-10 & 0,1683 & 0,6057 & 0,3926 \\
\hline Gesamt & 0,1644 & 0,5350 & 0,3544 \\
\hline
\end{tabular}

Tabelle 2: Deskriptive Statistik der Experimentdaten 
Frage 1: Wenn Sie nur ein einziges Mal mit ihrem Spielpartner zusammen spielen würden und bereits wüssten, wie er sich entschieden hat, wäre es für Sie vorteilhaft

\begin{tabular}{|l|c|c|c|}
\hline & $\begin{array}{c}\text { ohne } \\
\text { Kommunikation }\end{array}$ & $\begin{array}{c}\text { mit } \\
\text { Kommunikation }\end{array}$ & Gesamt \\
\hline \hline zu untertreiben. & 9 & 6 & 15 \\
& $(23,68 \%)$ & $(15,00 \%)$ & $(19,23 \%)$ \\
\hline wahrheitsgemäß & 24 & 20 & 44 \\
zu melden. & $(63,16 \%)$ & $(50,00 \%)$ & $(56,41 \%)$ \\
\hline zu übertreiben. & 5 & 14 & 19 \\
& $(13,16 \%)$ & $(35,00 \%)$ & $78,36 \%)$ \\
\hline & 38 & 40 & $(100 \%)$ \\
\hline
\end{tabular}

Frage 2: Wenn Sie Ihre Entscheidung bereits getroffen hätten und nicht mehr ändern könnten, wäre es für Sie vorteilhaft, wenn Ihr Spielpartner

\begin{tabular}{|l|c|c|c|}
\hline & $\begin{array}{c}\text { ohne } \\
\text { Kommunikation }\end{array}$ & $\begin{array}{c}\text { mit } \\
\text { Kommunikation }\end{array}$ & Gesamt \\
\hline \hline untertreibt. & 2 & 4 & 6 \\
& $(5,26 \%)$ & $(10,00 \%)$ & $(7,69 \%)$ \\
\hline wahrheitsgemäß & 15 & 12 & 27 \\
meldet. & $(39,47 \%)$ & $(30,00 \%)$ & $(34,62 \%)$ \\
\hline übertreibt. & 21 & 24 & 45 \\
& $(55,26 \%)$ & $(60,00 \%)$ & 78 \\
& 38 & 40 & $(100 \%)$ \\
\hline
\end{tabular}

Frage 3 (nur Kommunikation): Hat die Möglichkeit zur Absprache mit Ihrem Mitspieler Ihre Meldung beeinflusst?

\begin{tabular}{|c|c|c|}
\hline \hline Ja & Nein & Gesamt \\
\hline \hline 31 & 9 & 40 \\
$(77,5 \%)$ & $(22,5 \%)$ & $(100 \%)$ \\
\hline
\end{tabular}

Frage 4 (nur Kommunikation): Für den Fall, dass Ihnen Ihr Spielpartner seine Produktivität während der Kommunikationsphase mitgeteilt hat: Sind Sie davon ausgegangen, dass diese Produktivität seiner tatsächlichen Produktivität entsprach?

\begin{tabular}{|c|c|c|c|}
\hline \hline Ja & Nein & Keine Absprache & Gesamt \\
\hline 29 & 3 & 8 & 40 \\
$(72,50 \%)$ & $(7,50 \%)$ & $(20,00 \%)$ & $(100 \%)$ \\
\hline
\end{tabular}

Frage 5 (nur Kommunikation): Falls Sie sich in der letzten Runde abgesprochen haben: Fühlten Sie sich durch die Absprache gebunden?

\begin{tabular}{|c|c|c|c|}
\hline \hline Ja & Nein & Keine Absprache & Gesamt \\
\hline 27 & 8 & 5 & 40 \\
$(67,50 \%)$ & $(20,00 \%)$ & $(12,50 \%)$ & $(100 \%)$ \\
\hline
\end{tabular}

Tabelle 3: Auswertung des Fragebogens 


\begin{tabular}{|c|c|c|c|c|c|c|c|c|c|c|}
\hline & $\mid$ & 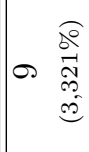 & 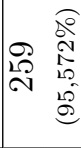 & 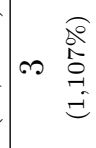 & 공 & & 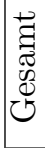 & 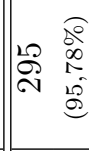 & 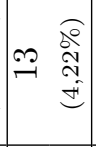 & 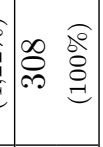 \\
\hline & 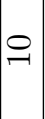 & 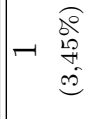 & 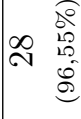 & 0 总 & 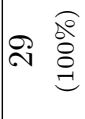 & & 으 & 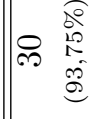 & 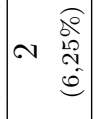 & $\because 0$ \\
\hline & o. & $\mid \begin{array}{ll} & \widehat{c} \\
0 & 1 \\
0 & 0 \\
0 & 0 \\
& 0\end{array}$ & 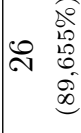 & 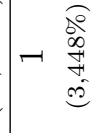 & 尺 $\stackrel{\overparen{D}}{\stackrel{\circ}{\Xi}}$ & & $\sigma$ & 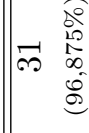 & 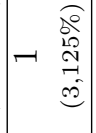 & $\therefore$ \\
\hline & $\infty$ & - & 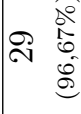 & $0 \stackrel{\circ}{0}$ & 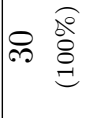 & & $\infty$ & $\mid \begin{array}{cc}0 & 0 \\
0 & 1 \\
\infty & 0 \\
0 & 8\end{array}$ & 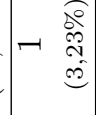 & $\rightarrow \infty$ \\
\hline & 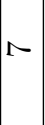 & 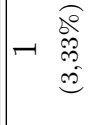 & 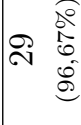 & $0 \stackrel{\circ}{0}$ & ه & & 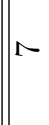 & 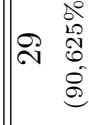 & $\infty \begin{array}{c}0 \\
0 \\
\infty \\
0 \\
0 \\
0 \\
0\end{array}$ & $\therefore$ \\
\hline & 0 & $0 \stackrel{\mathrm{g}}{\mathrm{g}}$ & 趈 & $0 \stackrel{\circ}{0}$ & 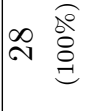 & & 0 & $\mid \begin{array}{cc}0 & 0 \\
0 & 1 \\
\infty & 0 \\
0 & 0\end{array}$ & 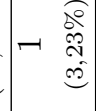 & बr \\
\hline 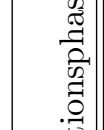 & 10 & 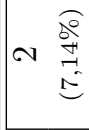 & 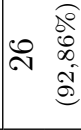 & $0 \stackrel{\circ}{0}$ & 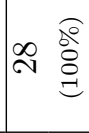 & & 10 & $\approx \stackrel{0}{\circ}$ & $0 \stackrel{\circ}{9}$ & $\approx 0$ \\
\hline 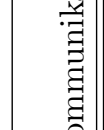 & + & 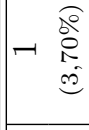 & 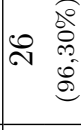 & 0 d & 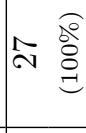 & & P & 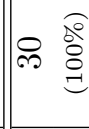 & 0 बㅇㅇㅇ & $\Leftrightarrow \stackrel{0}{\circ}$ \\
\hline 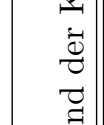 & $\infty$ & 0 बे & 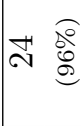 & $-\widehat{\stackrel{D}{+}}$ & 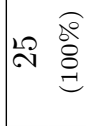 & ( & $\infty$ & 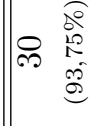 & 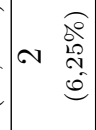 & $\approx 0$ \\
\hline 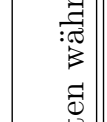 & N & 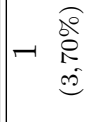 & 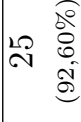 & $-\begin{array}{l}\widehat{0} \\
0 \\
0 \\
0\end{array}$ & 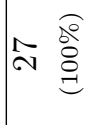 & 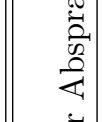 & | & 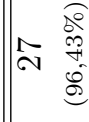 & 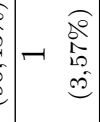 & 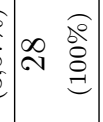 \\
\hline 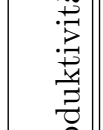 & - & 0 बㅇㅇㅇ & 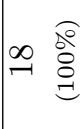 & $0 \stackrel{0}{0}$ & $\infty \stackrel{0}{\rightarrow} \stackrel{0}{\circ}$ & 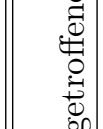 & - & 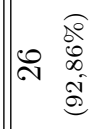 & $\sim>$ & 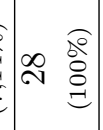 \\
\hline 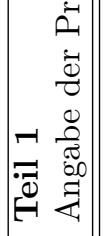 & 吾 & 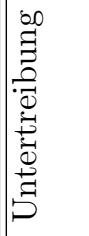 & 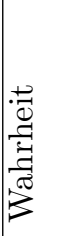 & 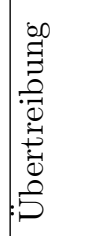 & 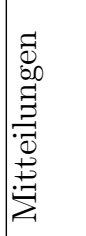 & 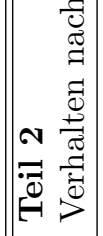 & $\mid$ & 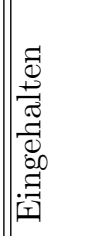 & 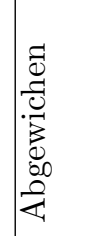 & 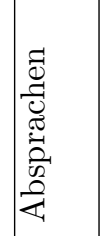 \\
\hline
\end{tabular}




\begin{tabular}{|l|c|c|c|}
\hline \multicolumn{2}{|l|}{} & $\begin{array}{c}\text { ohne } \\
\text { Kommunikation }\end{array}$ & $\begin{array}{c}\text { mit } \\
\text { Kommunikation }\end{array}$ \\
\hline \hline Übertreibung & $\Delta_{A}$ & 0,1700 & 0,2250 \\
& $\Delta_{R}$ & 0,3970 & 0,5787 \\
\hline \hline wahrheitsgemäße & $\Delta_{A}$ & 0,0667 & 0,2210 \\
Berichterstattung & $\Delta_{R}$ & 0,1883 & 0,5324 \\
\hline \hline Untertreibung & $\Delta_{A}$ & $-0,0289$ & 0,1700 \\
& $\Delta_{R}$ & $-0,0911$ & 0,4416 \\
\hline
\end{tabular}

Tabelle 5: Durchschnittliche absolute und relative Abweichung in Abhängigkeit der Beantwortung von Frage 1 des Fragebogens 